\title{
Labour market dynamics and worker heterogeneity during the Great Recession - Evidence from Europe
}

\author{
Ronald Bachmann ${ }^{1,2^{*}}$, Peggy Bechara ${ }^{1}$, Anica Kramer ${ }^{1,3}$ and Sylvi Rzepka ${ }^{1,3}$
}

\author{
* Correspondence: \\ bachmann@rwi-essen.de \\ ${ }^{1}$ Rheinisch-Westfälisches Institut für \\ Wirtschaftsforschung, \\ Hohenzollernstr. 1-3, 45131 Essen, \\ Germany \\ ${ }^{2}$ Forschungsinstitut zur Zukunft der \\ Arbeit (Institute for the Study of \\ Labor), Bonn, Germany \\ Full list of author information is \\ available at the end of the article
}

\begin{abstract}
Using harmonised micro data, this paper investigates the effects of the early phase (2008-10) of the recent economic crisis on transitions between labour market states in Europe. Our analysis focuses on individual heterogeneity, on the type of employment contract, and on cross-country differences. Our analysis shows that specific worker groups, such as men and young persons, were particularly strongly hit by the crisis. Furthermore, more transitions from employment, especially temporary employment to unemployment, were the main factor behind rising unemployment. Reduced unemployment outflows did not contribute substantially to the increase in unemployment during the early phase of the crisis.

JEL classification: J6, E24

Keywords: Recession; Labour market transitions; Markov transition matrices; Worker heterogeneity
\end{abstract}

\section{Introduction}

The recent financial and economic crisis led to high and persistent unemployment rates in all European labour markets. The initial impact of this economic shock on European labour markets was very strong: Between 2008 and 2010, four million persons lost their jobs within the European Monetary Union (ECB - European Central Bank 2012). Evidence from aggregate data suggests that specific worker groups were particularly strongly hit by the crisis, namely men and young persons. In addition, the type of employment contract seems to have had an important influence on the labour market effects of the economic crisis, which becomes particularly evident when comparing France, a country with very few fixed-term contracts, with Spain, where the opposite is the case. As a consequence, unemployment rose by much more in Spain than in France (Bentolila et al. 2012).

The contribution of flows in and out of unemployment to the cyclicality of unemployment has attracted a great deal of attention in the analysis of labour market dynamics (Shimer 2012). Recent articles have mainly found a relatively equal contribution of inflows and outflows to the unemployment stock (Elsby et al. 2009; Fujita and Ramey 2009). Fujita and Ramey (2009) as well as Fujita (2011) find evidence for differences in the timing of these effects, with the effect of the inflow rate being more prevalent during the early phase of a recession and the effect of the outflow rate being 
more important in the middle of a downturn. Yet, these studies have generally focussed on the US labour market and relied on aggregate data, thus neglecting potential composition effects, i.e. differences in the socio-demographic structure of the employed and unemployed. However, especially when including several countries into the analysis, it is important to control for composition effects.

In this study, we perform a micro-data based analysis of the labour market transitions in a large number of European countries and investigate how these transitions have been affected by the recent financial and economic crisis during its early phase (2008-2010). For this time period, the exogenous shock exerted by the economic and financial crisis can be assumed to be relatively similar across countries. After 2011, by contrast, the economic development was much more divergent across Europe, making it much more difficult to conceptually isolate differences in shocks from differences in labour market reactions. Therefore, focusing on the years 2008-2010 yields a clear picture of the labour market reactions to the initial impact of the Great Recession.

In doing so, we contribute to the literature in several ways. First, we give a general overview of the effects of the Great Recession on labour market dynamics in Europe and provide evidence on heterogeneous effects based on individual-level data. In doing so, we update Ward-Warmedinger and Macchiarelli (2014), who analyse labour market transitions in the EU before the Great Recession. Second, we show the importance of temporary and permanent contracts for labour market dynamics during the recession in a large number of European countries-previous studies focused on comparisons of a small number of countries (Bentolila et al. 2012). Third, our analysis of labour market transitions reveals the driving forces for the increase in unemployment during the Great Recession. ${ }^{1}$ Finally, given that the EU-SILC data are internationally comparable, we provide evidence on cross-country differences in labour market reactions to the crisis in Europe.

The paper is structured as follows: The next section briefly reviews the relevant literature. Section 3 presents the micro data set used as well as our empirical strategy. Section 4 contains the empirical evidence. The final section summarises our main results and concludes.

\section{Related literature}

The aggregate effects of the Great Recession are clearly established, particularly the effect on job loss and unemployment, as mentioned in the introduction (e.g. ECB - European Central Bank 2012). In the following, we briefly summarise previous findings on heterogeneous effects (age, gender, and contract types), especially with respect to labour market transitions.

The situation of young workers has attracted special attention, as it has become apparent that young workers have suffered disproportionately during the Great Recession (Bell and Blanchflower 2011). This is particularly worrying since evidence on past recessions demonstrates a substantial and long-lived negative influence of labour market entry in a recession on future wages and employment outcomes. This is, for example, shown by von Wachter and Bender (2008) in their analysis of the labour market history of German workers, and for West Germany as a whole by Bachmann et al. (2010). The latter authors find that labour market entrants earning less than the average starting wage are more likely to change their job as well as their occupation. Moreover, although job mobility tends to reduce the effects of labour market entry conditions, 
implying that job mobility operates as an adjustment mechanism that mitigates entry wage differentials, this process tends to take quite a long time. Finally, they show that these results hold not only for high-skilled, but also for medium-skilled and unskilled workers. Similar results are shown by Kahn (2010) for the US, where wages decrease by $6 \%$ with a $1 \%$ increase in the unemployment rate at graduation. Moreover, this effect persists for up to 15 years after graduation. Using Canadian data, Oreopoulos et al. (2012) show that the unemployment rate at year of graduation has negative effects on graduate earnings that last up to 10 years.

Labour market mobility generally differs between men and women; hence, one can expect heterogeneous effects of the crisis in this respect too. For example, using panel data from six European countries, Theodossiou and Zangelidis (2009) find that loweducated women are more likely to exit to non-employment than high-educated women and men of all education levels. With respect to the business cycle, lesseducated males display a pro-cyclical response of job-to-non-employment transitions, less-educated females a counter-cyclical response. Thus, judging from previous recessions, one should expect a decrease of transitions to non-employment for less-educated men, and an increase for less-educated women. For the recent recession, Verick (2009) finds that in OECD countries, young men were hit hardest, which is also due to men working in heavily impacted sectors such as construction.

Concerning contract types, there is some evidence for selected countries that the prevalence of temporary contracts had an important influence for labour markets during the crisis. This issue has been made obvious by a comparison of the performance of the French and Spanish labour markets (Bentolila et al. 2012). Before the Great Recession, temporary employment grew strongly in Spain, which led to a strong growth in overall employment, while the French labour market was relatively stagnant. In the recession, Bentolila et al. (2012) argue, temporary employment in Spain collapsed, which resulted in a large reduction in overall employment and a correspondingly large increase in unemployment. Therefore, the prevalence of temporary contracts before the crisis influenced the reaction of the labour market during the crisis.

The degree to which inflows and outflows determine unemployment has been strongly debated in the literature. This has typically been addressed by the analysis of aggregate time series of labour market transitions, especially for the US labour market. In this context, early studies such as Darby et al. (1986) found inflows into unemployment to be the decisive factor. Later studies found a more important role for outflows (Hall 2005; Shimer 2012). By now, a consensus seems to have been reached that the role of flows in and out of unemployment are relatively evenly split (Elsby et al. 2009; Yashiv 2008; Fujita and Ramey 2009). However, there are important differences between countries in this respect: Petrongolo and Pissarides (2008) find that in Spain and the UK, both inflows and outflows play an important role, whereas the outflow rate is the predominant factor in France. Elsby et al. (2013) argue that in Continental Europe, such a decomposition exercise should take into account that unemployment in these countries often deviates from its steady-state value. Examining a sample of 14 OECD countries, they find that unemployment outflows play a much more important role for unemployment variation than unemployment inflows in Anglo-Saxon countries; whereas for Continental European and Nordic countries, their relative importance is roughly equal. Furthermore, they find that increases in unemployment inflows 
precede a higher unemployment stock, whereas outflows lag behind increases in unemployment.

\section{Micro data and empirical strategy}

Our empirical analysis is mainly based on the European Union Statistics on Income and Living Conditions (EU-SILC), which provide representative and internationally comparable data on employment, income, poverty and living conditions for all EU Member States ${ }^{2}$ as well as Norway and Iceland, countries of the European Free Trade Association (EFTA). The data are collected at a yearly frequency and processed by Eurostat with the aim to harmonise the information and to ensure comparability across countries.

In order to be able to identify labour market transitions at an individual level, we use the longitudinal files of EU-SILC. The data sets consist of a four-year rotational panel, except for France (9-year panel), Norway (8-year panel), and Luxembourg (yearly panel) (Iacovou et al. 2012). This means that each person selected into the sample is interviewed for four years, and each subsequent year one quarter of all respondents is replaced by new respondents. This structure enables us to follow individuals up to a maximum of four consecutive years.

Each year, the longitudinal file available from Eurostat only contains those respondents that were interviewed both in the survey year and in the preceding year. In order to construct a data set with as many observations as possible, we combine the longitudinal files for 2005 to 2010. That is, the different longitudinal data sets are merged together, resulting in a data set that covers the time period from 2004 to 2010. We follow Engel and Schaffner (2012) in order to adjust the weighting scheme of the micro data accordingly. As a consequence, our weighted sample is representative for the population of the countries under consideration. For most countries, EU-SILC data are available for the whole time period covering the years 2004 to 2010. For a subset of countries, the survey was first conducted in 2005 (Cyprus, Czech Republic, Hungary, Lithuania, Poland, Slovenia, Slovakia and the United Kingdom) and thus data for 2004 are not available. For Bulgaria and Malta the years 2004 and 2005 are not covered since their survey started in 2006. In addition, no data are available for Ireland in 2010. EU-SILC data for Germany only cover the years 2005 and 2006, for Romania only the years from 2007. We therefore exclude these two countries from the regression analysis. However, we compute descriptive statistics for these two countries using micro data from the European Union Labour Force Survey (EU-LFS).

In the empirical analyses we concentrate on labour market adjustments through the extensive margin, i.e. the transitions across different labour market states. It is commonly asserted that the extensive margin of labour adjustment is significantly more important than the intensive margin (cf. King and Rebelo 1999, Langot and Quintero-Rojas 2008, Merkl and Wesselbaum 2011). ${ }^{3}$ In order to construct labour market transitions, we combine the information on the economic status of an individual in a given year with the information on the labour market status in the preceding year; this allows computing yearly transitions between those states. In addition, the data set includes information on the type of contract held at the time of the interview, which allows us to identify transitions between employment with a permanent or temporary contract and other labour market states. ${ }^{4}$ 
Our empirical analysis focuses on labour market transitions from the origin states employment and unemployment. We therefore estimate multinomial logit models for these two labour market states. The destination states considered are (dependent) employment (in a further step, we also differentiate between temporary and permanent employment), self-employment, unemployment, education, and inactivity. The general form of the predicted probability from the multinomial logit model can be written as

$$
\operatorname{Pr}(y=m \mid \boldsymbol{X})=\frac{\exp \left(\boldsymbol{X}^{\prime} \beta_{m \mid b}\right)}{\sum_{j=1}^{J} \exp \left(\boldsymbol{X}^{\prime} \beta_{j \mid b}\right)}, \text { with } \mathrm{m}=1, \ldots, \mathbf{j},
$$

where $\mathrm{y}$ is one of the $\mathbf{j}$ labour market states, $\mathrm{b}$ is the base category, and $\mathbf{X}$ is a vector of explanatory variables. When we take being employed $(E)$ as the base category, the predicted probability of being unemployed $(\mathrm{U})$ is:

$$
\operatorname{Pr}(y=U \mid \boldsymbol{X})=\frac{\exp \left(\boldsymbol{X}^{\prime} \beta_{U \mid E}\right)}{\exp \left(\boldsymbol{X}^{\prime} \beta_{U \mid E}\right)+\sum_{j=2}^{J} \exp \left(\boldsymbol{X}^{\prime} \beta_{j \mid b}\right)}
$$

The vector of explanatory variables includes individual characteristics, namely gender, age (dummy variables for belonging to the age groups 15-24, 25-34, 35-54 and 55-64), marital status, level of education (dummy variables for low, medium and high levels of education), full-time or part-time work (only for origin state employment), and occupation dummies (only for transitions out of employment ${ }^{5}$ ). We also include the following household characteristics: Number of children (younger than 5 years, between 5 and 14 years), number of persons aged 15-64, number of persons older than 65 in the household, as well as the presence of a partner in the household, and the labour market status of the partner. The regressions also include country fixed effects in order to control for region-specific factors that are constant over time.

As the main focus of the analysis is on the impact of the financial and economic crisis on labour market dynamics, the vector of explanatory variables also includes an indicator variable for the economic crisis. This variable takes the value of 0 for the pre-crisis period and 1 for the crisis period, which started in a specific quarter in 2008 or 2009. In order to define the start of the recession for each country separately, we use quarterly GDP data from Eurostat and define a recession as at least two consecutive quarters of negative GDP growth. ${ }^{6}$ Note that our indicator therefore captures the overall effect of the slowdown in aggregate economic activity on labour market dynamics. We apply the crisis indicator to the individual-level observations in EU-SILC by taking into account the interview date of each individual (available on a quarterly basis) and the timing of the crisis in each country. Interacting the crisis indicator with demographic characteristics (gender, age, level of education) allows us to investigate how the impact of the crisis varies over demographic groups.

\section{Empirical evidence}

\subsection{Labour market transitions and worker heterogeneity}

In the years before the crisis, the overall unemployment rate of our country sample displayed a downward trend and amounted to $9.6 \%$ in 2008 (see Fig. 1). With the onset of the crisis, the unemployment rate increased to $12.1 \%$ in 2009 and $13 \%$ in 2010 . It 


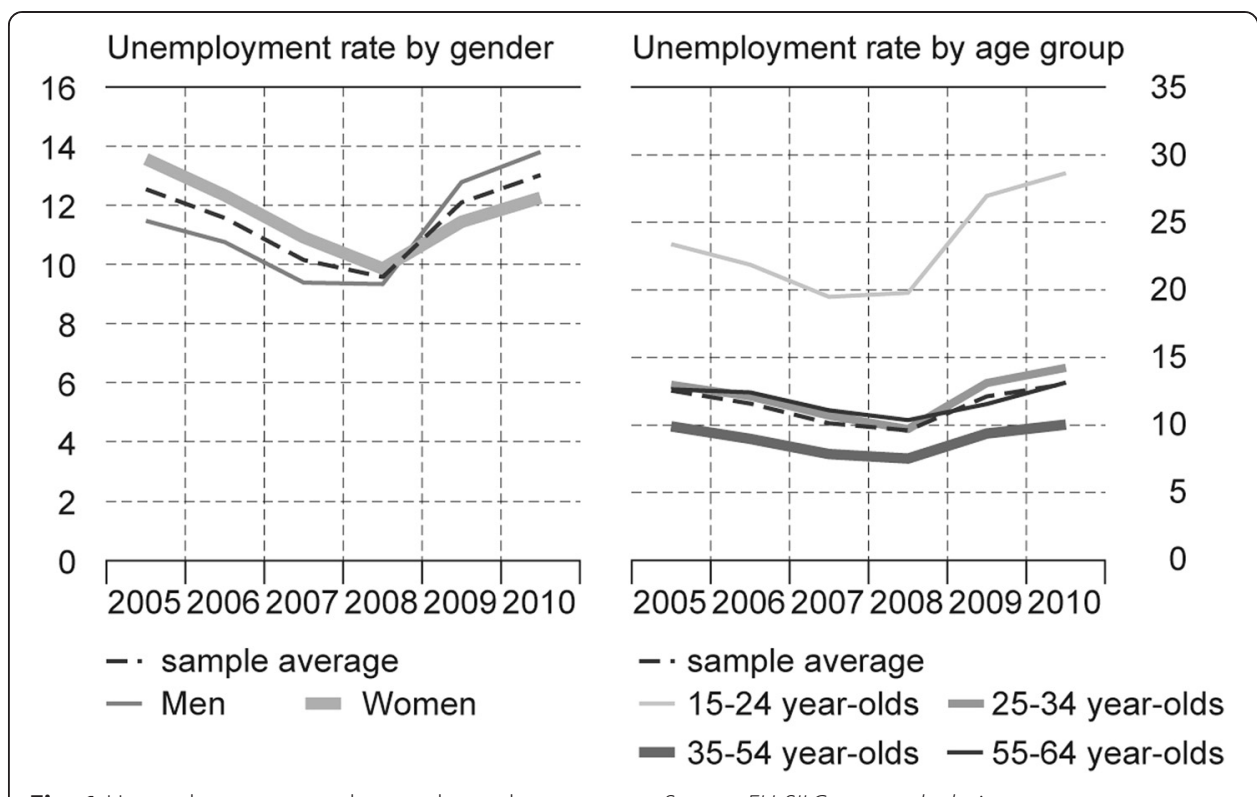

Fig. 1 Unemployment rates by gender and age groups. Source: EU-SILC, own calculations

also becomes apparent that the crisis marks a turning point in the development of the unemployment rates of men and women. Before the crisis, women had higher unemployment rates than men, but this gender difference declined strongly in 2008. After the beginning of the crisis, the unemployment rate of men increased much more than that of women (13.8\% versus $12.2 \%$ in 2010$)$.

The crisis also had heterogeneous effects with respect to age groups. The unemployment rate of young workers (15-24 years) is generally higher than the unemployment rates of other age groups, but it increased particularly strongly during the crisis, reaching $28.6 \%$ for our country sample in 2010 (Fig. 1). The unemployment rate of the 25-34 and 55-65 year-olds is very close to the average unemployment rate over the time period considered. In contrast, the prime-age group (35-54 years) displays consistently lower unemployment than the average unemployment rate, but follows the same trend.

In order to analyse which transitions changed most strongly as the European economies went into recession, we compute Markov transition matrices between labour market states for the time periods before and during the crisis (Table 1). ${ }^{7}$ This shows that before, 91.9\% of those employed in a given year were still employed in the following year; however, this rate drops to $90.8 \%$ during the crisis. This drop is mainly due to an increase of the transition rate from employment-to-unemployment of 1 percentage point because the transitions from employment to other labour market states remain largely unchanged.

As for transitions from unemployment, we observe a decrease of the yearly transition rate to employment from $27.6 \%$ to $24.9 \%$ and a corresponding increase of the rate at which the unemployed remained in this labour market state of $1.1 \%$. Furthermore, we observe increases in the transition rates to education and to inactivity.

The transition rates from the other labour market states change to a smaller extent. Most notably, the transition rate from self-employment to employment decreases from $6.6 \%$ to $5.8 \%$, the transition rate from education to employment declines from 15.3\% to $13.5 \%$, and the transition rate from inactivity to employment falls from $5.0 \%$ to 
Table 1 Yearly Markov transition matrix for all countries

\begin{tabular}{|c|c|c|c|c|c|c|c|c|c|c|}
\hline \multirow[t]{3}{*}{ Origin } & \multicolumn{10}{|l|}{ Destination } \\
\hline & Employment & Self-employment & Unemployment & Education & Inactivity & Employment & Self-employment & Unemployment & Education & Inactivity \\
\hline & \multicolumn{5}{|l|}{ Pre-crisis } & \multicolumn{5}{|l|}{ During the crisis } \\
\hline Employment & 91.9 & 1.4 & 2.9 & 0.6 & 3.2 & 90.8 & 1.5 & 3.9 & 0.6 & 3.3 \\
\hline Self-employment & 6.6 & 87.4 & 1.6 & 0.2 & 4.2 & 5.8 & 87.5 & 2.1 & 0.3 & 4.3 \\
\hline Unemployment & 27.6 & 3.4 & 51.5 & 1.9 & 15.5 & 24.9 & 3.3 & 52.6 & 2.8 & 16.3 \\
\hline Education & 15.3 & 0.8 & 3.8 & 77.0 & 3.0 & 13.5 & 0.9 & 5.5 & 77.4 & 2.8 \\
\hline Inactivity & 5.0 & 1.4 & 2.3 & 0.6 & 90.7 & 4.5 & 1.4 & 2.7 & 0.7 & 90.6 \\
\hline Total & 51.4 & 8.9 & 5.9 & 7.7 & 26.2 & 51.4 & 9.3 & 6.5 & 7.6 & 25.1 \\
\hline
\end{tabular}


4.5\%. While these transition rates are crucial for the evolution of employment, they are not directly linked to the evolution of the unemployment rate, our focus of analysis. In the following, we therefore concentrate on transitions emanating from employment and from unemployment.

In order to find out whether the changes in transition rates between the pre-crisis and the crisis periods are statistically significant, we run the multinomial regression models described in Section 3, focussing on the flows out of employment and unemployment. Table 2 presents our baseline regression results for the transitions out of employment. Our first main finding is that during the crisis, employment stability decreased significantly and sizably-by 1.1 percentage points-and flows from employment to unemployment increased significantly by 1.3 percentage points. For other transition destinations-besides employment and unemployment-the coefficient of the crisis indicator is insignificant. Apparently, the early phase of the crisis did not have strong effects on these other transitions.

Besides this overall picture on the changes during the economic crisis, our regression results indicate important heterogeneities between demographic groups. With respect to gender, the results for the pre-crisis period show that men are 1.5 percentage points more likely to remain employed than women, and they are less likely to become unemployed (0.5 percentage points - Table 3$)$. However, this picture eroded during the economic crisis, as employment stability of men was affected more by the crisis than that of women. In particular, men were nearly 1 percentage point less likely to stay in employment during the crisis than women. In a similar vein, employmentto-unemployment flows increased for men by nearly 1 percentage point more than for women during the crisis.

Turning to different age groups, we find that before the crisis, the chances of remaining in employment were highest for those aged 35-54 and lowest for those aged 55-65 (Table 4). The transition rate from employment to unemployment is higher for the youngest cohort than for those aged 35-54 (the reference group). The coefficients on the interaction terms with the crisis indicator suggest that the youngest cohort has been hit particularly strongly by the economic crisis: The employment-to-unemployment transition rate increases by 0.5 percentage points more for those aged 15-24 than the transition rate of the reference group.

With respect to skill groups, our regression results show that before the crisis, highskilled workers find it easier to remain in employment and have a lower transition rate into unemployment than the reference group, the medium-skilled (Table 5). During the economic crisis, employment stability is reduced by 1 percentage point for medium-skilled workers, the reference group. Our regression results do not suggest any differences by skill groups in this context, i.e. the reduction of employment stability is equally pronounced. A similar picture emerges for flows from employment to unemployment: The crisis increases the corresponding transition rates, but we do not observe any differences by skill level.

We now turn to an analysis of the transitions out of unemployment. Table 6 shows the general effect of the crisis: The transition rate from unemployment to employment decreases by about 4 percentage points, which is statistically significant at the $5 \%$ level. This is in line with previous empirical studies as that of the ECB - European Central Bank (2012). Somewhat surprisingly, the econometric analysis does not suggest higher persistence of unemployment since the coefficient of the crisis indicator for staying 
Table 2 Yearly transitions from employment to different labour market states, regression results

\begin{tabular}{|c|c|c|c|c|c|}
\hline & $\mathrm{EE}$ & ES & EU & EEd & $\mathrm{El}$ \\
\hline \multirow[t]{2}{*}{ Crisis indicator } & $-0.0114^{c}$ & -0.0005 & $0.0125^{c}$ & 0.0000 & -0.0006 \\
\hline & $(0.0026)$ & $(0.001)$ & $(0.0024)$ & $(0.0001)$ & $(0.0013)$ \\
\hline Female & $\begin{array}{l}\text { Reference } \\
\text { category }\end{array}$ & $\begin{array}{l}\text { Reference } \\
\text { category }\end{array}$ & $\begin{array}{l}\text { Reference } \\
\text { category }\end{array}$ & $\begin{array}{l}\text { Reference } \\
\text { category }\end{array}$ & $\begin{array}{l}\text { Reference } \\
\text { category }\end{array}$ \\
\hline \multirow[t]{2}{*}{ Male } & $0.0110^{c}$ & $0.0058^{c}$ & -0.0019 & 0.0000 & $-0.0150^{c}$ \\
\hline & $(0.0035)$ & $(0.001)$ & $(0.0016)$ & $(0.0001)$ & $(0.0021)$ \\
\hline \multirow[t]{2}{*}{ Age $15-24$} & $-0.0540^{c}$ & 0.0010 & $0.0223^{c}$ & $0.0167^{c}$ & $0.0139^{c}$ \\
\hline & (0.0033) & $(0.001)$ & $(0.003)$ & $(0.0016)$ & $(0.0036)$ \\
\hline \multirow[t]{2}{*}{ Age 25-34 } & $-0.0214^{c}$ & $0.0033^{c}$ & $0.0098^{c}$ & $0.0027^{c}$ & $0.0056^{c}$ \\
\hline & $(0.0032)$ & $(0.001)$ & $(0.0025)$ & $(0.0005)$ & (0.0019) \\
\hline Age $35-54$ & $\begin{array}{l}\text { Reference } \\
\text { category }\end{array}$ & $\begin{array}{l}\text { Reference } \\
\text { category }\end{array}$ & $\begin{array}{l}\text { Reference } \\
\text { category }\end{array}$ & $\begin{array}{l}\text { Reference } \\
\text { category }\end{array}$ & $\begin{array}{l}\text { Reference } \\
\text { category }\end{array}$ \\
\hline \multirow[t]{2}{*}{ Age 55-65 } & $-0.0990^{c}$ & -0.0001 & -0.0008 & $-0.0011^{c}$ & $0.1009^{c}$ \\
\hline & $(0.0152)$ & $(0.0007)$ & $(0.0017)$ & $(0.0002)$ & $(0.0148)$ \\
\hline Single & $\begin{array}{l}\text { Reference } \\
\text { category }\end{array}$ & $\begin{array}{l}\text { Reference } \\
\text { category }\end{array}$ & $\begin{array}{l}\text { Reference } \\
\text { category }\end{array}$ & $\begin{array}{l}\text { Reference } \\
\text { category }\end{array}$ & $\begin{array}{l}\text { Reference } \\
\text { category }\end{array}$ \\
\hline \multirow[t]{2}{*}{ Married } & $0.0026^{\mathrm{b}}$ & -0.0005 & $-0.0069^{c}$ & $-0.0005^{b}$ & $0.0053^{c}$ \\
\hline & $(0.0012)$ & $(0.0012)$ & $(0.0011)$ & $(0.0002)$ & $(0.0009)$ \\
\hline \multirow[t]{2}{*}{ Low skilled (ISCED 0-2) } & $-0.0168^{c}$ & 0.0006 & $0.0103^{c}$ & $-0.0003^{c}$ & $0.0063^{c}$ \\
\hline & $(0.002)$ & $(0.0006)$ & $(0.0011)$ & $(0.0001)$ & $(0.0008)$ \\
\hline Medium skilled (ISCED 3-4) & $\begin{array}{l}\text { Reference } \\
\text { category }\end{array}$ & $\begin{array}{l}\text { Reference } \\
\text { category }\end{array}$ & $\begin{array}{l}\text { Reference } \\
\text { category }\end{array}$ & $\begin{array}{l}\text { Reference } \\
\text { category }\end{array}$ & $\begin{array}{l}\text { Reference } \\
\text { category }\end{array}$ \\
\hline \multirow[t]{2}{*}{ High skilled (ISCED 5) } & $0.0096^{c}$ & $-0.0015^{\mathrm{a}}$ & $-0.004^{c}$ & 0.0000 & $-0.0042^{c}$ \\
\hline & $(0.0018)$ & $(0.0009)$ & $(0.0009)$ & $(0.0001)$ & $(0.0012)$ \\
\hline \multirow{2}{*}{$\begin{array}{l}\text { Number of children }(<=4) \text { in } \\
\text { household }\end{array}$} & $-0.0067^{c}$ & 0.0006 & 0.0001 & $-0.0004^{c}$ & $0.0065^{c}$ \\
\hline & $(0.0017)$ & $(0.0005)$ & $(0.0006)$ & $(0.0001)$ & $(0.0017)$ \\
\hline \multirow{2}{*}{$\begin{array}{l}\text { Number of children (5-14) in } \\
\text { household }\end{array}$} & -0.0005 & $0.0011^{c}$ & $0.0013^{c}$ & 0.0001 & $-0.002^{c}$ \\
\hline & $(0.0007)$ & $(0.0003)$ & $(0.0005)$ & $(0)$ & $(0.0005)$ \\
\hline \multirow{2}{*}{$\begin{array}{l}\text { Number of employable persons } \\
(15-64) \text { in household }\end{array}$} & $0.0016^{c}$ & 0.0002 & 0.0003 & $0.0002^{b}$ & $-0.0022^{c}$ \\
\hline & $(0.0006)$ & $(0.0002)$ & $(0.0003)$ & $(0.0001)$ & $(0.0004)$ \\
\hline \multirow{2}{*}{$\begin{array}{l}\text { Number of elderly }(>=65) \text { in } \\
\text { household }\end{array}$} & $-0.0044^{c}$ & 0.0004 & 0.0009 & 0.0000 & $0.0031^{c}$ \\
\hline & $(0.0015)$ & $(0.0004)$ & $(0.0008)$ & $(0.0001)$ & $(0.0008)$ \\
\hline \multirow{2}{*}{$\begin{array}{l}\text { Full-time employed partner in } \\
\text { household }\end{array}$} & $0.0111^{c}$ & 0.0001 & $-0.0063^{c}$ & $-0.0002^{b}$ & $-0.0047^{b}$ \\
\hline & $(0.0032)$ & $(0.0004)$ & $(0.0014)$ & $(0.0001)$ & $(0.0022)$ \\
\hline \multirow{2}{*}{$\begin{array}{l}\text { Part-time employed partner in } \\
\text { household }\end{array}$} & $0.0132^{c}$ & -0.0006 & $-0.0066^{c}$ & $-0.0003^{c}$ & $-0.0056^{c}$ \\
\hline & $(0.0026)$ & $(0.0008)$ & $(0.0016)$ & $(0.0001)$ & (0.0019) \\
\hline $\begin{array}{l}\text { Inactive/unemployed partner in } \\
\text { household }\end{array}$ & $\begin{array}{l}\text { Reference } \\
\text { category }\end{array}$ & $\begin{array}{l}\text { Reference } \\
\text { category }\end{array}$ & $\begin{array}{l}\text { Reference } \\
\text { category }\end{array}$ & $\begin{array}{l}\text { Reference } \\
\text { category }\end{array}$ & $\begin{array}{l}\text { Reference } \\
\text { category }\end{array}$ \\
\hline \multirow[t]{2}{*}{ No partner in household } & $0.0057^{\mathrm{b}}$ & 0.0004 & 0.0005 & $0.001^{c}$ & $-0.0076^{c}$ \\
\hline & $(0.0024)$ & $(0.0013)$ & $(0.001)$ & $(0.0002)$ & $(0.0022)$ \\
\hline
\end{tabular}


Table 2 Yearly transitions from employment to different labour market states, regression results (Continued)

\begin{tabular}{|c|c|c|c|c|c|}
\hline Full-time employed & $\begin{array}{l}\text { Reference } \\
\text { category }\end{array}$ & $\begin{array}{l}\text { Reference } \\
\text { category }\end{array}$ & $\begin{array}{l}\text { Reference } \\
\text { category }\end{array}$ & $\begin{array}{l}\text { Reference } \\
\text { category }\end{array}$ & $\begin{array}{l}\text { Reference } \\
\text { category }\end{array}$ \\
\hline \multirow[t]{2}{*}{ Part-time employed } & $-0.0382^{c}$ & $0.0071^{c}$ & $0.0126^{c}$ & $0.0029^{c}$ & $0.0156^{c}$ \\
\hline & $(0.0023)$ & $(0.0015)$ & $(0.0027)$ & $(0.0006)$ & $(0.0019)$ \\
\hline Occupation dummies & included & included & included & included & included \\
\hline Country dummies & included & included & included & included & included \\
\hline Pseudo-R-squared & 0.1094 & & & & \\
\hline Observations & 578,331 & & & & \\
\hline
\end{tabular}

EU-SILC, own calculations. Multinomial logit model. ${ }^{\mathrm{a}} /^{\mathrm{b}} / \mathrm{c}$ : statistically significant at least at the 10\%-/5\%-/1\%-level. - The model includes transitions between the following labour market states: employment (E) (i.e. persons remaining in employment), unemployment (U), self-employment (S), education (Ed) and inactivity (I). - Robust standard errors clustered at the country level in parentheses

unemployed is not statistically significant. This also applies to the other destination states. This is a first indication that higher inflow rates from employment into unemployment, rather than reduced outflow rates from unemployment, brought about the increased unemployment rates in the early phase of the financial and economic crisis.

We now look at heterogeneities of the crisis impact on transitions out of unemployment by gender, age, and skill group. Before the crisis, men had a 6 percentage point higher transition rate from unemployment to employment (Table 7). During the Great Recession, men are 3.5 percentage points less likely to make a transition to employment than women. Therefore, while transitions from unemployment to employment did not play an important role in the aggregate, they do explain to some extent why unemployment rose more strongly during the crisis for men than for women.

As for differences between age groups, we see a clear pattern of higher unemploymentto-employment transitions for younger ages before the crisis (Table 8). Those aged between 15 and 24 (between 25 and 34), on average, had an 11 percentage points (8.3 percentage points) higher chance of becoming employed compared to the prime age group of those aged 35 to 54 .

Table 3 Yearly transitions from employment to different labour market states, gender-specific effects of the crisis

\begin{tabular}{lll}
\hline & $E$ & $U$ \\
\hline Female & Reference category & Reference category \\
Male & $0.0147^{c}$ & $-0.0058^{c}$ \\
& $(0.0044)$ & $(0.0021)$ \\
Crisis indicator & $-0.0071^{c}$ & $0.0074^{c}$ \\
& $(0.0027)$ & $(0.0014)$ \\
Crisis ${ }^{c}$ Male & $-0.0083^{\mathrm{a}}$ & $0.0088^{c}$ \\
Other individual covariates & included & included \\
Occupation dummies & included & included \\
Country dummies & included & included \\
Pseudo-R-squared & 0.1097 & \\
Observations & 578,331 &
\end{tabular}

EU-SILC, own calculations. Multinomial logit model. ${ }^{a} / \mathrm{b} / \mathrm{c}$ : statistically significant at least at the $10 \%-/ 5 \%-/ 1 \%$-level. - The model includes transitions between the following labour market states: employment (E) (i.e. persons remaining in employment), unemployment (U), self-employment (S), education (Ed) and inactivity (I); only the first two are presented. - Robust standard errors clustered at the country level in parentheses 
Table 4 Yearly transitions from employment to different labour market states, age group-specific effects of the crisis

\begin{tabular}{|c|c|c|}
\hline & E & U \\
\hline \multirow[t]{2}{*}{ Age 15-24 } & $-0.0489^{c}$ & $0.0188^{\mathrm{c}}$ \\
\hline & $(0.0043)$ & $(0.0029)$ \\
\hline \multirow[t]{2}{*}{ Age 25-34 } & $-0.0203^{c}$ & $0.0098^{c}$ \\
\hline & $(0.0036)$ & $(0.0027)$ \\
\hline Age $35-54$ & Reference category & Reference category \\
\hline \multirow[t]{2}{*}{ Age $55-65$} & $-0.0992^{c}$ & 0.0006 \\
\hline & $(0.0164)$ & $(0.0028)$ \\
\hline \multirow[t]{2}{*}{ Crisis indicator } & $-0.0099^{c}$ & $0.0119^{c}$ \\
\hline & $(0.0023)$ & $(0.0021)$ \\
\hline \multirow[t]{2}{*}{ Crisis $^{*}$ Age $15-24$} & -0.0077 & $0.0049^{b}$ \\
\hline & $(0.0049)$ & $(0.0022)$ \\
\hline \multirow[t]{2}{*}{ Crisis $^{*}$ Age $25-34$} & -0.0022 & 0.0000 \\
\hline & $(0.0021)$ & $(0.0012)$ \\
\hline \multirow[t]{2}{*}{ Crisis $^{*}$ Age $55-65$} & -0.0009 & -0.0027 \\
\hline & $(0.0042)$ & $(0.002)$ \\
\hline Other individualcovariates & included & included \\
\hline Occupation dummies & included & included \\
\hline Country dummies & included & included \\
\hline Pseudo-R-squared & 0.1095 & \\
\hline Observations & 578,331 & \\
\hline
\end{tabular}

EU-SILC, own calculations. Multinomial logit model. ${ }^{a} /^{b} / c$ : statistically significant at least at the $10 \%-/ 5 \%-/ 1 \%$-level. - The model includes transitions between the following labour market states: employment (E) (i.e. persons remaining in employment), unemployment (U), self-employment (S), education (Ed) and inactivity (I); only the first two are presented. - Robust standard errors clustered at the country level in parentheses

In contrast, near-retirement ages have an 18.7 percentage points lower transition rate into employment. Considering the impact of the crisis, we only detect a significantly stronger negative impact for the 25 to 34-year-olds compared to the prime-age group. Therefore, the negative impact on young workers, which has been much discussed, does not seem to be driven by the evolution of the transition rate from unemployment to employment.

As for skill groups, we observe that prior to the crisis, low-skilled workers display a 7.5 percentage points lower transition rate from unemployment to employment than medium-skilled persons, while high-skilled workers have a 7.7 percentage points higher rate (see Table 9). During the crisis, the high-skilled experience a higher chance of remaining in unemployment than the medium-skilled, indicating a more persistent unemployment for this worker group.

\subsection{The role of contract type for labour market transitions}

As discussed in Section 2, the type of contract a worker holds plays an important role for labour market dynamics. Given our large sample of European countries, we can provide a broader perspective and thereby add to the selected results of Bentolila et al. (2012). We therefore split aggregate employment into permanent and temporary employment, and compute the corresponding Markov transition matrix for the time 
Table 5 Yearly transitions from employment to different labour market states, skill groupspecificeffects of the crisis

\begin{tabular}{lll}
\hline & $E$ & $U$ \\
\hline Low skilled (ISCED 0-2) & $-0.0174^{c}$ & $0.0097^{c}$ \\
Medium skilled (ISCED 3-4) & $(0.0026)$ & $(0.0016)$ \\
High skilled (ISCED 5) & Reference category & Reference category \\
& $0.0106^{c}$ & $-0.0047^{c}$ \\
Crisis indicator & $(0.0023)$ & $(0.001)$ \\
& $-0.0113^{c}$ & $0.0118^{c}$ \\
Crisis ${ }^{*}$ Low skilled & $(0.0027)$ & $(0.0014)$ \\
& 0,0017 & 0,0010 \\
Crisis ${ }^{*}$ High skilled & $(0.0027)$ & $(0.0034)$ \\
& $-0,0023$ & 0,0015 \\
Other individualcovariates & $(0.0024)$ & $(0.002)$ \\
Occupation dummies & included & included \\
Country dummies & included & included \\
Pseudo-R-squared & included & included \\
Observations & 0,1095 & \\
\hline
\end{tabular}

EU-SILC, own calculations. Multinominal logit model. a $/ \mathrm{b} / \mathrm{c}$ : statistically significant at least at the $10 \%-/ 5 \%-/ 1 \%$-level. - The model includes transitions between the following labour market states: employment (E) (i.e. persons remaining in employment), unemployment $(\mathrm{U})$, self-employment $(\mathrm{S})$, education $(\mathrm{Ed})$ and inactivity $(\mathrm{I})$; only the first two are presented. - Robust standard errors clustered at country level in parentheses

periods before and during the crisis (Table 10). ${ }^{8}$ We begin our analysis with flows from employment.

For Europe as a whole, employment stability declined more strongly for those workers holding a temporary contract than for those with a permanent contract. While the rate at which workers remained employed (either permanent or temporary) from one year to the next declined from 93.3 to $92.3 \%$ for permanent workers, it decreased from 82 to $78.2 \%$ for temporary workers. Furthermore, during the Great Recession, the transition rate from temporary employment to unemployment increased by more than that of permanent employment to unemployment. Compared to the pre-crisis period, during the crisis the transition rate to unemployment increased by 4.1 percentage points for temporary workers and by 1.3 percentage points for permanent workers. Finally, during the crisis the transitions from temporary employment to permanent employment declined strongly, i.e. temporary employment was much less of a stepping stone to permanent employment during the crisis than during the pre-crisis period.

These findings are generally confirmed by the econometric evidence, which reveals a significant increase in the transition rate from permanent employment to unemployment of 1.02 percentage points and an also significant but quantitatively much more important increase in the transition rate from temporary employment to unemployment of 4.85 percentage points (Table 11). The regression results also show that, when controlling for composition effects, employment stability did not decline significantly during the recession for both permanent employment and temporary employment. This result for temporary employment may appear surprising at first glance. However, it should be noted that the outflow rate from temporary employment, which mirrors its stability, mainly consists of the transition rates to permanent employment and to unemployment. 
Table 6 Yearly transitions from unemployment to different labour market states

\begin{tabular}{|c|c|c|c|c|c|}
\hline & UU & UE & US & UEd & UI \\
\hline \multirow[t]{2}{*}{ Crisis indicator } & 0.0466 & $-0.0401^{b}$ & -0.0030 & 0.0018 & -0.0053 \\
\hline & $(0.0301)$ & $(0.0167)$ & $(0.0031)$ & $(0.0013)$ & $(0.0125)$ \\
\hline Female & $\begin{array}{l}\text { Reference } \\
\text { category }\end{array}$ & $\begin{array}{l}\text { Reference } \\
\text { category }\end{array}$ & $\begin{array}{l}\text { Reference } \\
\text { category }\end{array}$ & $\begin{array}{l}\text { Reference } \\
\text { category }\end{array}$ & $\begin{array}{l}\text { Reference } \\
\text { category }\end{array}$ \\
\hline \multirow[t]{2}{*}{ Male } & $0.0258^{\mathrm{a}}$ & $0.0488^{c}$ & $0.0226^{c}$ & $-0.0022^{b}$ & $-0.095^{c}$ \\
\hline & $(0.0149)$ & $(0.0135)$ & $(0.0008)$ & $(0.0009)$ & $(0.0084)$ \\
\hline \multirow[t]{2}{*}{ Age 15-24 } & $-0.1019^{c}$ & $0.1024^{c}$ & $-0.0125^{c}$ & $0.0578^{c}$ & $-0.0458^{c}$ \\
\hline & $(0.0233)$ & $(0.014)$ & $(0.0039)$ & $(0.0061)$ & $(0.0102)$ \\
\hline \multirow[t]{2}{*}{ Age 25-34 } & $-0.0619^{c}$ & $0.076^{c}$ & -0.0016 & $0.0171^{c}$ & $-0.0298^{c}$ \\
\hline & (0.0139) & $(0.012)$ & $(0.0018)$ & $(0.0033)$ & $(0.0069)$ \\
\hline Age $35-54$ & $\begin{array}{l}\text { Reference } \\
\text { category }\end{array}$ & $\begin{array}{l}\text { Reference } \\
\text { category }\end{array}$ & $\begin{array}{l}\text { Reference } \\
\text { category }\end{array}$ & $\begin{array}{l}\text { Reference } \\
\text { category }\end{array}$ & $\begin{array}{l}\text { Reference } \\
\text { category }\end{array}$ \\
\hline \multirow[t]{2}{*}{ Age 55-65 } & 0.0238 & $-0.1885^{c}$ & $-0.0157^{c}$ & $-0.01^{c}$ & $0.1904^{c}$ \\
\hline & $(0.0172)$ & $(0.0212)$ & $(0.0025)$ & $(0.0022)$ & $(0.0206)$ \\
\hline Single & $\begin{array}{l}\text { Reference } \\
\text { category }\end{array}$ & $\begin{array}{l}\text { Reference } \\
\text { category }\end{array}$ & $\begin{array}{l}\text { Reference } \\
\text { category }\end{array}$ & $\begin{array}{l}\text { Reference } \\
\text { category }\end{array}$ & $\begin{array}{l}\text { Reference } \\
\text { category }\end{array}$ \\
\hline \multirow[t]{2}{*}{ Married } & -0.0045 & -0.0058 & -0.0016 & $-0.0033^{c}$ & $0.0151^{c}$ \\
\hline & $(0.0095)$ & $(0.0064)$ & $(0.0025)$ & $(0.001)$ & $(0.0055)$ \\
\hline \multirow[t]{2}{*}{ Low skilled (ISCED 0-2) } & $0.0692^{c}$ & $-0.0725^{c}$ & $-0.0113^{c}$ & $-0.0049^{c}$ & $0.0195^{c}$ \\
\hline & $(0.0154)$ & $(0.0141)$ & $(0.0014)$ & $(0.0008)$ & $(0.0039)$ \\
\hline Medium skilled (ISCED 3-4) & $\begin{array}{l}\text { Reference } \\
\text { category }\end{array}$ & $\begin{array}{l}\text { Reference } \\
\text { category }\end{array}$ & $\begin{array}{l}\text { Reference } \\
\text { category }\end{array}$ & $\begin{array}{l}\text { Reference } \\
\text { category }\end{array}$ & $\begin{array}{l}\text { Reference } \\
\text { category }\end{array}$ \\
\hline \multirow[t]{2}{*}{ High skilled (ISCED 5) } & $-0.0577^{c}$ & $0.069^{c}$ & $0.0175^{c}$ & $0.0041^{c}$ & $-0.0329^{c}$ \\
\hline & $(0.0139)$ & $(0.0135)$ & $(0.0038)$ & $(0.0014)$ & $(0.0044)$ \\
\hline \multirow{2}{*}{$\begin{array}{l}\text { Number of children }(<=4) \text { in } \\
\text { household }\end{array}$} & $-0.0132^{c}$ & $-0.018^{c}$ & 0.0004 & $-0.0025^{a}$ & $0.0332^{c}$ \\
\hline & $(0.0044)$ & $(0.0049)$ & $(0.0031)$ & $(0.0013)$ & $(0.0031)$ \\
\hline \multirow{2}{*}{$\begin{array}{l}\text { Number of children (5-14) in } \\
\text { household }\end{array}$} & -0.0015 & 0.0006 & -0.0001 & 0.0006 & 0.0004 \\
\hline & $(0.0033)$ & $(0.0037)$ & $(0.0014)$ & $(0.0006)$ & $(0.0024)$ \\
\hline \multirow{2}{*}{$\begin{array}{l}\text { Number of employable persons } \\
\text { (15-64) in household }\end{array}$} & $0.0093^{\mathrm{a}}$ & 0.0010 & -0.0003 & 0.0003 & $-0.0103^{c}$ \\
\hline & $(0.0055)$ & $(0.0041)$ & $(0.0006)$ & $(0.0003)$ & $(0.0033)$ \\
\hline \multirow{2}{*}{$\begin{array}{l}\text { Number of elderly }(>=65) \text { in } \\
\text { household }\end{array}$} & $0.016^{b}$ & $-0.0233^{c}$ & -0.0005 & 0.0008 & $0.0071^{c}$ \\
\hline & $(0.008)$ & $(0.0073)$ & $(0.0019)$ & $(0.0014)$ & $(0.0025)$ \\
\hline \multirow{2}{*}{$\begin{array}{l}\text { Full-time employed partner in } \\
\text { household }\end{array}$} & $-0.0594^{b}$ & 0.0398 & 0.0036 & 0.0005 & $0.0155^{b}$ \\
\hline & $(0.0253)$ & $(0.0264)$ & $(0.0043)$ & $(0.0015)$ & $(0.0069)$ \\
\hline \multirow{2}{*}{$\begin{array}{l}\text { Part-time employed partner in } \\
\text { household }\end{array}$} & $-0.0487^{a}$ & $0.0568^{a}$ & $0.01^{a}$ & $-0.0069^{c}$ & -0.0112 \\
\hline & $(0.0285)$ & $(0.0313)$ & $(0.0054)$ & $(0.0012)$ & $(0.0144)$ \\
\hline $\begin{array}{l}\text { Inactive/unemployed partner in } \\
\text { household }\end{array}$ & $\begin{array}{l}\text { Reference } \\
\text { category }\end{array}$ & $\begin{array}{l}\text { Reference } \\
\text { category }\end{array}$ & $\begin{array}{l}\text { Reference } \\
\text { category }\end{array}$ & $\begin{array}{l}\text { Reference } \\
\text { category }\end{array}$ & $\begin{array}{l}\text { Reference } \\
\text { category }\end{array}$ \\
\hline No partner in household & $0.0395^{b}$ & -0.0167 & $-0.0133^{c}$ & 0.0021 & -0.0116 \\
\hline
\end{tabular}


Table 6 Yearly transitions from unemployment to different labour market states (Continued)

\begin{tabular}{|c|c|c|c|c|c|}
\hline & $(0.0175)$ & $(0.0127)$ & $(0.0047)$ & $(0.0028)$ & $(0.0086)$ \\
\hline Pseudo-R-squared & 0.086 & & & & \\
\hline Observations & 69,281 & & & & \\
\hline
\end{tabular}

The net effect on the outflow rate was insignificant, because of offsetting effects: the transition rate to permanent employment significantly declined, and the transition rate to unemployment significantly increased. Hence, the crisis did not significantly affect the stability of temporary employment.

The regression results also point to strong gender differences with respect to the contract type of employment. For instance, men are 0.5 percentage points less likely to remain in permanent employment than women during the crisis; for the latter, the crisis has no significant impact. Furthermore, the crisis increases flows from permanent employment to unemployment especially for men, since their rate of becoming unemployed out of permanent employment increases by 0.6 percentage points more than for women (Table 12). However, this picture changes when we focus on temporary employment as state of origin. The transition rate from temporary employment to unemployment increases by 4.2 percentage points more for men than for women (see Table 13). This means that the strong effect of the crisis on men is to a large extent triggered by the upsurge in transitions from temporary employment to unemployment.

As for heterogeneous effects for age groups, we observe only few statistically significant different impacts of the crisis by age (Table 14). On the one hand, the crisis decreases the rate at which workers remain in permanent employment by 1 percentage point for the youngest age group, signaling a substantial reduction in permanent job stability. On the other hand, the oldest age group is marginally less likely (by 0.3 percentage points) to become unemployed out of permanent employment during the crisis than the reference

Table 7 Yearly transitions from unemployment to different labour market states, gender-specific effects of the crisis

\begin{tabular}{lll}
\hline & $U$ & $E$ \\
\hline Male & 0.0053 & $0.0612^{c}$ \\
& $(0.0137)$ & $(0.0124)$ \\
Crisis indicator & 0.0182 & -0.0193 \\
& $(0.0277)$ & $(0.0158)$ \\
Crisis ${ }^{*}$ Male & $0.0534^{c}$ & $-0.035^{c}$ \\
& $(0.0074)$ & $(0.0051)$ \\
Other individual covariates & included & included \\
Country dummies & included & included \\
Year dummies & included & included \\
Pseudo-R-squared & 0.0863 & \\
Observations & 69,281 &
\end{tabular}

EU-SILC, own calculations. Multinomial logit model. ${ }^{\mathrm{a}} / \mathrm{b} / \mathrm{c}$ : statistically significant at least at the 10\%-/5\%-/1\%-level. Transitions take place between the following labour market states: employment (E), unemployment (U), self-employment (S), education (Ed) and inactivity (I); only the first two are presented. - Robust standard errors clustered at country level in parentheses 
Table 8 Yearly transitions from unemployment to different labour market states, age group effects of the crisis

\begin{tabular}{|c|c|c|}
\hline & U & E \\
\hline \multirow[t]{2}{*}{ Age 15-24 } & $-0.1039^{c}$ & $0.1099^{c}$ \\
\hline & $(0.028)$ & $(0.0154)$ \\
\hline \multirow[t]{2}{*}{ Age 25-34 } & $-0.0683^{c}$ & $0.0829^{c}$ \\
\hline & $(0.0174)$ & $(0.0112)$ \\
\hline Age $35-54$ & Reference category & Reference category \\
\hline \multirow[t]{2}{*}{ Age $55-65$} & 0.0227 & $-0.1872^{c}$ \\
\hline & $(0.0218)$ & $(0.0225)$ \\
\hline \multirow[t]{2}{*}{ Crisis indicator } & 0.0390 & $-0.031^{a}$ \\
\hline & $(0.0326)$ & $(0.0174)$ \\
\hline \multirow[t]{2}{*}{ Crisis $^{*}$ Age $15-24$} & 0.0005 & -0.0193 \\
\hline & $(0.0191)$ & $(0.0127)$ \\
\hline \multirow[t]{2}{*}{ Crisis $^{*}$ Age $25-34$} & 0.0175 & $-0.0163^{b}$ \\
\hline & $(0.0116)$ & $(0.0071)$ \\
\hline \multirow[t]{2}{*}{ Crisis $^{*}$ Age $55-65$} & 0.0091 & -0.0049 \\
\hline & $(0.0215)$ & $(0.019)$ \\
\hline Other individual covariates & included & included \\
\hline Country dummies & included & included \\
\hline Year dummies & included & included \\
\hline Pseudo-R-squared & 0.0862 & \\
\hline Observations & 69,281 & \\
\hline
\end{tabular}

EU-SILC, own calculations. Multinomial logit model. ${ }^{a} /^{\mathrm{b}} / \mathrm{c}^{\mathrm{c}}$ : statistically significant at least at the 10\%-/5\%-/1\%-level. Transitions take place between the following labour market states: employment (E), unemployment (U), self-employment (S), education (Ed) and inactivity (I); only the first two are presented. - Robust standard errors clustered at country level in parentheses

group. However, this picture changes if we look at temporary employment as state of origin. In general, the older age cohorts are by 5 percentage points less likely to remain in temporary employment than the middle-aged group. Furthermore, in the crisis the temporary-employment-to-unemployment transition rate for 15 to 24-year-olds was nearly 3 percentage points higher than the transition rate of the 35 to 54-year-olds (Table 15).

We observe a similar pattern for skill groups, i.e. there are no major changes for transitions out of permanent employment during the crisis (Table 16). The crisis does not negatively affect the rate at which the low-skilled remain in permanent employment but does negatively impact the medium-skilled workers. However, for temporary employment as state of origin, we find that the transition probabilities of the high-skilled workers were affected most by the crisis (Table 17). Flows from temporary employment to unemployment increase by up to 2.8 percentage points more for high-skilled than for medium skilled workers.

Turning to labour market flows out of unemployment, the descriptive statistics reveal that the transition rate from unemployment to permanent employment declined by 2.7 percentage points, the transition rate to temporary employment by 1.5 percentage points (Table 10). Interestingly, the regression results reveal that while the decrease of the transition rate from unemployment to permanent is significant, the decline in the transition rate from unemployment to temporary employment is not (Table 11). Therefore, temporary employment at least did not lose its job-creating role even in the time 
Table 9 Yearly transitions from unemployment to different labour market states, skill group-specific effects of the crisis

\begin{tabular}{lll}
\hline & $U$ & $E$ \\
\hline Low skilled (ISCED 0-2) & $0.0555^{c}$ & $-0.0749^{c}$ \\
Medium skilled (ISCED 3-4) & $(0.0204)$ & $(0.0153)$ \\
High skilled (ISCED 5) & Reference category & Reference category \\
& $-0.077^{c}$ & $0.0767^{c}$ \\
Crisis indicator & $(0.0139)$ & $(0.0124)$ \\
& 0.0246 & $-0.0395^{c}$ \\
Crisis ${ }^{*}$ Low skilled & $(0.0217)$ & $(0.0129)$ \\
& 0.0320 & 0.0065 \\
Crisis ${ }^{*}$ High skilled & $(0.0315)$ & $(0.0199)$ \\
& $0.0483^{\mathrm{b}}$ & -0.0178 \\
Other individual covariates & $(0.0206)$ & $(0.0135)$ \\
Country dummies & included & included \\
Year dummies & included & included \\
Pseudo-R-squared & included & included \\
Observations & 0.0864 & \\
\hline
\end{tabular}

EU-SILC, own calculations. Multinomial logit model. ${ }^{\mathrm{a}} /{ }^{\mathrm{b}} / \mathrm{c}$ : statistically significant at least at the 10\%-/5\%-/1\%-level. Transitions take place between the following labour market states: employment (E), unemployment (U), self-employment (S), education (Ed) and inactivity (I); only the first two are presented. - Robust standard errors clustered at country level in parentheses

of the economic crisis and thus might potentially serve as one important instrument in order to re-build European labour markets. ${ }^{9}$

Looking at heterogeneous effects, the more pronounced effect of the crisis on men, which was shown above for transitions emanating from employment, also holds for transitions from unemployment to temporary employment, as Table 18 shows. The rate at which the unemployed find a temporary job drops by 1.8 percentage points for men; for women, this rate remains constant during the crisis.

Furthermore, for the different age groups, we do not discover any heterogeneous effects when disaggregating the flows from unemployment into temporary and permanent employment (Table 19). Finally, Table 20 suggests that highly skilled persons have been affected more by the economic crisis in one respect: Their transition rates into permanent employment decrease by 2.1 percentage points more than that of the medium-skilled group.

\subsection{Inflows vs. outflows and cross-country differences}

The previous descriptive and econometric evidence shows that the worker flows between employment and unemployment strongly changed during the crisis, while other labour market transitions were affected to a much smaller extent. Hence, the increase of the aggregate unemployment rate during the crisis was mainly driven by transitions from employment to unemployment and vice versa. Therefore, in the final step of the analysis, we compare the change in flows in and out of unemployment for the European countries in order to shed light on the countries' adjustment patterns to the crisis. For this, we use the descriptive evidence computed from EU-SILC and EU-LFS, which allows us to take into account the maximum number of countries (see Section 2). 
Table 10 Yearly Markov transition matrix for all countries, detailed employment states

\begin{tabular}{|c|c|c|c|c|c|c|c|c|c|c|c|c|}
\hline \multirow[t]{2}{*}{ Origin } & \multicolumn{12}{|l|}{ Destination } \\
\hline & $\begin{array}{l}\text { Perm } \\
\text { employment }\end{array}$ & $\begin{array}{l}\text { Temp } \\
\text { employment }\end{array}$ & $\begin{array}{l}\text { Self- } \\
\text { employment }\end{array}$ & Unemployment & Education & Inactivity & $\begin{array}{l}\text { Perm } \\
\text { employment }\end{array}$ & $\begin{array}{l}\text { Temp } \\
\text { employment }\end{array}$ & $\begin{array}{l}\text { Self- } \\
\text { employment }\end{array}$ & Unemployment & Education & Inactivity \\
\hline & \multicolumn{6}{|l|}{ Pre-crisis } & \multicolumn{6}{|c|}{ During the crisis } \\
\hline $\begin{array}{l}\text { Perm } \\
\text { Employment }\end{array}$ & 90.3 & 3.0 & 1.2 & 2.0 & 0.3 & 3.2 & 89.7 & 2.6 & 1.3 & 3.3 & 0.3 & 3.0 \\
\hline $\begin{array}{l}\text { Temp } \\
\text { employment }\end{array}$ & 27.2 & 54.8 & 2.1 & 9.8 & 2.0 & 4.1 & 23.1 & 55.1 & 2.2 & 13.9 & 1.8 & 3.9 \\
\hline $\begin{array}{l}\text { Self- } \\
\text { employment }\end{array}$ & 4.8 & 1.7 & 87 & 1.8 & 0.2 & 4.5 & 3.7 & 1.6 & 87.7 & 2.4 & 0.3 & 4.4 \\
\hline Unemployment & 11.8 & 16 & 3.7 & 51.9 & 2.2 & 14.5 & 9.1 & 14.5 & 3.6 & 56.7 & 3.0 & 13.1 \\
\hline Education & 6.2 & 7.2 & 0.9 & 4.9 & 77.7 & 3.2 & 3.9 & 5.6 & 1.0 & 6.8 & 79.7 & 3.0 \\
\hline Inactivity & 3.3 & 1.9 & 1.5 & 2.8 & 0.7 & 89.7 & 2.6 & 1.7 & 1.7 & 3.5 & 0.8 & 89.7 \\
\hline Total & 43.7 & 8.0 & 10.1 & 6.6 & 8.0 & 23.6 & 41.6 & 7.7 & 11.1 & 8.3 & 8.6 & 22.8 \\
\hline
\end{tabular}


Table 11 Crisis indicator for different labour market transitions, detailed employment states

\begin{tabular}{lllllll}
\hline & PermE & TempE & $S$ & $U$ & Ed & I \\
\hline PermE & -0.0016 & $-0.0055^{\mathrm{b}}$ & -0.0012 & $0.0102^{\mathrm{c}}$ & $0.0000^{\mathrm{a}}$ & $-0.002^{\mathrm{c}}$ \\
& $(0.0028)$ & $(0.0028)$ & $(0.001)$ & $(0.0015)$ & 0.0000 & $(0.0006)$ \\
TempE & $-0.0441^{c}$ & -0.0013 & -0.0016 & $0.0485^{\mathrm{b}}$ & 0.0003 & -0.0018 \\
& $(0.0158)$ & $(0.0109)$ & $(0.0013)$ & $(0.0194)$ & $(0.0004)$ & $(0.0015)$ \\
$\mathrm{U}$ & $-0.0225^{c}$ & -0.0158 & -0.0035 & 0.0468 & 0.0013 & -0.0063 \\
& $(0.0044)$ & $(0.016)$ & $(0.0034)$ & $(0.0335)$ & $(0.0013)$ & $(0.013)$ \\
\hline
\end{tabular}

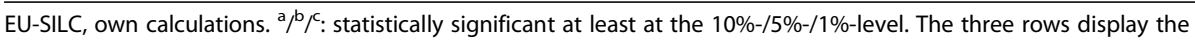
marginal effects on the crisis indicator from three separate multinomial logit models for the origin states permanent employment (PermE), temporary employment (TempE) and unemployment (U). - Robust standard errors clustered at country level in parentheses

The inflow rate into unemployment was much stronger than the decrease in the outflow rate (see Fig. 2). For the sample average, these growth rates amounted to 36\% and $-9.6 \%$, respectively. Furthermore, while the overwhelming majority of countries mirror this overall adjustment pattern, there was relatively strong heterogeneity in the degree by which countries reacted to the crisis. On the one side of the spectrum, there are those countries which were very strongly hit by the crisis and which feature the highest increase in unemployment inflows. These countries include the Baltic States, Ireland, and Spain. On the other side of the spectrum, countries such as Germany and Poland did not experience a strong recession, and therefore the flow rates did not change by much.

At least in some countries of the European Union, aggregate labour market dynamics are likely to be strongly influenced by the prevalence of temporary contracts. ${ }^{10} \mathrm{We}$ therefore analyse the flow rates from permanent and temporary employment to unemployment in more detail. For Europe as a whole, Fig. 3 shows that the increase in the transition rate from permanent employment to unemployment was actually higher $(+65 \%)$ than the increase in the transition rate from temporary employment to unemployment $(+42 \%)$. This aggregate figure hides strong country heterogeneity. On the

Table 12 Yearly transitions from permanent employment to different labour market states, gender-specific effects of the crisis

\begin{tabular}{llll}
\hline & PermE & TempE & $U$ \\
\hline Female & Reference category & Reference category & Reference category \\
Male & $0.0132^{c}$ & $-0.002^{\mathrm{a}}$ & $-0.0039^{\mathrm{a}}$ \\
& $(0.0049)$ & $(0.0011)$ & $(0.002)$ \\
Crisis indicator & 0.0014 & $-0.0055^{\mathrm{b}}$ & $0.0065^{\mathrm{c}}$ \\
& $(0.0031)$ & $(0.0024)$ & $(0.001)$ \\
Crisis ${ }^{*}$ Male & $-0.0055^{c}$ & -0.0001 & $0.0062^{c}$ \\
& $(0.002)$ & $(0.0024)$ & $(0.0015)$ \\
Other individual covariates & included & included & included \\
Occupation dummies & included & included & included \\
Country dummies & included & included & included \\
Pseudo-R-squared & 0.0925 & & \\
Observations & 402,731 & & \\
\hline
\end{tabular}

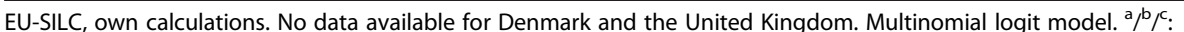
statistically significant at least at the $10 \%-/ 5 \%-/ 1 \%$-level. Transitions take place between the following labour market states: Permanent employment (PermE), temporary employment, (TempE) unemployment (U), self-employment (S), education (Ed) and inactivity (I); only the first three are presented. - Robust standard errors clustered at country level in parentheses 
Table 13 Yearly transitions from temporary employment to different labour market states, gender-specific effects of the crisis

\begin{tabular}{llll}
\hline & TempE & PermE & $U$ \\
\hline Female & Reference category & Reference category & Reference category \\
Male & 0.0033 & $0.0367^{c}$ & $-0.0256^{c}$ \\
& $(0.0223)$ & $(0.0134)$ & $(0.0076)$ \\
Crisis indicator & 0.0046 & $-0.0292^{\mathrm{a}}$ & $0.0249^{\mathrm{b}}$ \\
& $(0.0165)$ & $(0.0175)$ & $(0.0118)$ \\
Crisis ${ }^{*}$ Male & -0.0113 & -0.0271 & $0.0421^{\mathrm{b}}$ \\
& $(0.029)$ & $(0.0178)$ & $(0.0164)$ \\
Other individual covariates & included & included & included \\
Occupation dummies & included & included & included \\
Country dummies & included & included & included \\
Pseudo-R-squared & 0.0786 & & \\
Observations & 62,439 & & \\
\hline
\end{tabular}

EU-SILC, own calculations. No data available for Denmark and the United Kingdom. Multinomial logit model. ${ }^{\mathrm{a}} /^{\mathrm{b}} / \mathrm{c}$ : statistically significant at least at the 10\%-/5\%-/1\%-level. Transitions take place between the following labour market states: Permanent employment (PermE), temporary employment, (TempE) unemployment (U), self-employment (S), education (Ed) and inactivity (I); only the first three are presented. - Robust standard errors clustered at country level in parentheses

Table 14 Yearly transitions from permanent employment to different labour market states, age group-specific effects of the crisis

\begin{tabular}{|c|c|c|c|}
\hline & PermE & TempE & $U$ \\
\hline \multirow[t]{2}{*}{ Age 15-24 } & $-0.0785^{c}$ & $0.0415^{c}$ & $0.0142^{c}$ \\
\hline & $(0.0056)$ & $(0.0044)$ & $(0.0039)$ \\
\hline \multirow[t]{2}{*}{ Age 25-34 } & $-0.0345^{c}$ & $0.0154^{c}$ & $0.0075^{c}$ \\
\hline & $(0.0025)$ & $(0.0013)$ & $(0.002)$ \\
\hline Age 35-54 & Reference category & Reference category & Reference category \\
\hline \multirow[t]{2}{*}{ Age 55-65 } & $-0.1104^{c}$ & $-0.0071^{a}$ & 0.0050 \\
\hline & $(0.0136)$ & $(0.0037)$ & $(0.0043)$ \\
\hline \multirow[t]{2}{*}{ Crisis indicator } & -0.0014 & $-0.0056^{a}$ & $0.0106^{c}$ \\
\hline & $(0.0036)$ & $(0.003)$ & $(0.0016)$ \\
\hline \multirow[t]{2}{*}{ Crisis $^{*}$ Age $15-24$} & $-0.01^{c}$ & 0.0043 & 0.0036 \\
\hline & $(0.0034)$ & $(0.0027)$ & $(0.0023)$ \\
\hline \multirow[t]{2}{*}{ Crisis $^{*}$ Age $25-34$} & 0.0014 & -0.0010 & -0.0008 \\
\hline & $(0.0026)$ & $(0.0018)$ & $(0.001)$ \\
\hline \multirow[t]{2}{*}{ Crisis $^{*}$ Age $55-65$} & 0.0035 & -0.0018 & $-0.0034^{b}$ \\
\hline & $(0.003)$ & $(0.0023)$ & $(0.0016)$ \\
\hline Other individual covariates & included & included & included \\
\hline Occupation dummies & included & included & included \\
\hline Country dummies & included & included & included \\
\hline Pseudo-R-squared & 0.0924 & & \\
\hline Observations & 402,731 & & \\
\hline
\end{tabular}

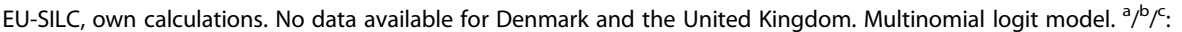
statistically significant at least at the 10\%-/5\%-/1\%-level. Transitions take place between the following labour market states: Permanent employment (PermE), temporary employment, (TempE) unemployment (U), self-employment (S), education (Ed) and inactivity (I); only estimates for the first three categories are presented. - Robust standard errors clustered at country level in parentheses 
Table 15 Yearly transitions from temporary employment to different labour market states, age group-specific effects of the crisis

\begin{tabular}{|c|c|c|c|}
\hline & TempE & PermE & U \\
\hline \multirow[t]{2}{*}{ Age 15-24 } & -0.0152 & 0.0075 & -0.0052 \\
\hline & $(0.0268)$ & $(0.0286)$ & $(0.0071)$ \\
\hline \multirow[t]{2}{*}{ Age $25-34$} & -0.0119 & 0.0108 & 0.0044 \\
\hline & $(0.0081)$ & $(0.0087)$ & $(0.009)$ \\
\hline Age $35-54$ & Reference category & Reference category & Reference category \\
\hline \multirow[t]{2}{*}{ Age 55-65 } & $-0.0459^{c}$ & $-0.0517^{c}$ & -0.0047 \\
\hline & $(0.0141)$ & $(0.0193)$ & $(0.012)$ \\
\hline \multirow[t]{2}{*}{ Crisis indicator } & 0.0077 & $-0.0387^{\mathrm{a}}$ & $0.0389^{b}$ \\
\hline & $(0.0109)$ & $(0.0213)$ & $(0.0191)$ \\
\hline \multirow[t]{2}{*}{ Crisis $^{*}$ Age $15-24$} & -0.0077 & -0.0302 & $0.0273^{b}$ \\
\hline & $(0.0323)$ & $(0.0264)$ & $(0.0135)$ \\
\hline \multirow[t]{2}{*}{ Crisis $^{*}$ Age $25-34$} & $-0.0176^{a}$ & -0.0006 & 0.0075 \\
\hline & $(0.009)$ & $(0.007)$ & $(0.0079)$ \\
\hline \multirow[t]{2}{*}{ Crisis $^{*}$ Age 55-65 } & -0.0188 & 0.0224 & 0.0024 \\
\hline & $(0.0158)$ & $(0.0194)$ & $(0.0095)$ \\
\hline Other individual covariates & included & included & Included \\
\hline Occupation dummies & included & included & Included \\
\hline Country dummies & included & included & Included \\
\hline Pseudo-R-squared & 0.0784 & & \\
\hline Observations & 62,439 & & \\
\hline
\end{tabular}

EU-SILC, own calculations. No data available for Denmark and the United Kingdom. Multinomial logit model. ${ }^{\mathrm{a}} / \mathrm{b} / \mathrm{c}$ : statistically significant at least at the 10\%-/5\%-/1\%-level. Transitions take place between the following labour market states: Permanent employment (PermE), temporary employment, (TempE) unemployment (U), self-employment (S), education (Ed) and inactivity (I); only estimates for the first three categories are presented. - Robust standard errors clustered at country level in parentheses

one hand, there are countries which experienced a strong increase in the transition rate from permanent employment to unemployment. These countries include the Baltic States and Ireland, which were strongly hit by the crisis and at the same time are characterised by relatively low employment protection (Martin and Scarpetta 2012, Muravyev A 2010).

On the other hand, there are countries which hardly experience any increase in the transition rate from permanent employment to unemployment, but a strong increase in the transition rate from temporary employment to unemployment. Examples are Austria, Cyprus and Sweden. Interestingly, Spain - which is often cited as the prime example of a dual labour market-only features a slightly higher increase in the transition rate from temporary employment to unemployment $(+102 \%)$ than from permanent employment to unemployment (+92\%). ${ }^{11}$ Although the difference between permanent and temporary contracts was not that large in Spain in relative terms (i.e. in terms of the rate of increase of the transition rate), the large prevalence of temporary contracts nevertheless implied a very strong increase in the absolute number of transitions from employment to unemployment, which is in line with evidence presented by Eichhorst et al. (2010), Bentolila et al. (2012), and Silva and Vázquez-Grenno (2013).

While temporary contracts play an important role for labour market dynamics during the Great Recession in a number of countries, the reasons for cross-country differences 
Table 16 Yearly transitions from permanent employment to different labour market states, skill group-specific effects of the crisis

\begin{tabular}{llll}
\hline & PermE & TempE & $U$ \\
\hline Low skilled ISCED 0-2 & $-0.021^{c}$ & $0.0064^{\mathrm{b}}$ & $0.0075^{\mathrm{c}}$ \\
Medium skilled ISCED 3-4 & $(0.0025)$ & $(0.0026)$ & $(0.0019)$ \\
High skilled ISCED 5 & Reference category & Reference category & Reference category \\
& $0.0048^{\mathrm{a}}$ & $0.0029^{\mathrm{b}}$ & -0.0025 \\
Crisis indicator & $(0.0025)$ & $(0.0013)$ & $(0.0017)$ \\
& $-0.0041^{\mathrm{a}}$ & $-0.0031^{\mathrm{a}}$ & $0.0109^{\mathrm{c}}$ \\
Crisis ${ }^{*}$ Low skilled & $(0.0024)$ & $(0.0017)$ & $(0.0015)$ \\
& $0.0054^{\mathrm{a}}$ & -0.0046 & -0.0012 \\
Crisis ${ }^{*}$ High skilled & $(0.0032)$ & $(0.0037)$ & $(0.0025)$ \\
& 0.0030 & -0.0046 & -0.0009 \\
Other individual covariates & $(0.0019)$ & $(0.0029)$ & $(0.0035)$ \\
Occupation dummies & included & included & included \\
Country dummies & included & included & included \\
Pseudo-R-squared & 0.0925 & included & included \\
Observations & 402,731 & & \\
\hline
\end{tabular}

EU-SILC, own calculations. No data available for Denmark and the United Kingdom. Multinomial logit model. a $/ \mathrm{b} / \mathrm{c}$ : statistically significant at least at the $10 \%-/ 5 \%-/ 1 \%$-level. Transitions take place between the following labour market states: Permanent employment (PermE), temporary employment, (TempE) unemployment (U), self-employment (S), education (Ed) and inactivity (I); only estimates for the first three categories are presented. - Robust standard errors clustered at country level in parentheses

Table 17 Yearly transitions from temporary employment to different labour market states, skill group-specific effects of the crisis

\begin{tabular}{|c|c|c|c|}
\hline & TempE & PermE & $U$ \\
\hline \multirow[t]{2}{*}{ Low skilled (ISCED 0-2) } & 0.0123 & $-0.0387^{c}$ & $0.0224^{c}$ \\
\hline & $(0.0141)$ & $(0.0089)$ & $(0.0077)$ \\
\hline Medium skilled (ISCED 3-4) & Reference category & Reference category & Reference category \\
\hline \multirow[t]{2}{*}{ High skilled (ISCED 5) } & $0.0234^{b}$ & $0.0239^{b}$ & $-0.036^{c}$ \\
\hline & $(0.0092)$ & $(0.0114)$ & $(0.0074)$ \\
\hline \multirow[t]{2}{*}{ Crisis indicator } & 0.0050 & $-0.0391^{c}$ & $0.0309^{b}$ \\
\hline & $(0.0125)$ & $(0.011)$ & $(0.0126)$ \\
\hline \multirow[t]{2}{*}{ Crisis ${ }^{*}$ Low skilled } & -0.0147 & -0.0024 & 0.0301 \\
\hline & $(0.0109)$ & $(0.0225)$ & $(0.0239)$ \\
\hline \multirow[t]{2}{*}{ Crisis ${ }^{*}$ High skilled } & -0.0063 & $-0.0169^{b}$ & $0.0278^{c}$ \\
\hline & $(0.0093)$ & $(0.0084)$ & $(0.0102)$ \\
\hline Other individual covariates & included & included & included \\
\hline Occupation dummies & included & included & included \\
\hline Country dummies & included & included & included \\
\hline Pseudo-R-squared & 0.0785 & & \\
\hline Observations & 62,439 & & \\
\hline
\end{tabular}

EU-SILC, own calculations. No data available for Denmark and the United Kingdom. Multinomial logit model. a $/ \mathrm{b} / \mathrm{c}$ : statistically significant at least at the $10 \%-/ 5 \%-/ 1 \%$-level. Transitions take place between the following labour market states: Permanent employment (PermE), temporary employment, (TempE) unemployment (U), self-employment (S), education (Ed) and inactivity (I); only estimates for the first three categories are presented. - Robust standard errors clustered at country level in parentheses 
Table 18 Yearly transitions from unemployment to different labour market states (detailed employment states), gender-specific effects of the crisis

\begin{tabular}{llll}
\hline & $U$ & PermE & TempE \\
\hline Female & Reference category & Reference category & Reference category \\
Male & 0.0012 & $0.032^{c}$ & $0.0289^{c}$ \\
& $(0.0118)$ & $(0.0041)$ & $(0.0081)$ \\
Crisis indicator & 0.0212 & $-0.0151^{c}$ & -0.0053 \\
& $(0.0308)$ & $(0.0052)$ & $(0.0169)$ \\
Crisis $^{*}$ Male & $0.0493^{c}$ & $-0.0127^{c}$ & $-0.0175^{c}$ \\
& $(0.0068)$ & $(0.0044)$ & $(0.0036)$ \\
Other individual covariates & included & included & included \\
Country dummies & included & included & included \\
Year dummies & included & included & included \\
Pseudo-R-squared & 0.0845 & & \\
Observations & 65,872 & & \\
\hline EU-SLC, own calculion & & &
\end{tabular}

EU-SILC, own calculations. No data available for Denmark and the United Kingdom. Multinomial logit model. ${ }^{\mathrm{a}} / \mathrm{b} / \mathrm{c}$ : statistically significant at least at the $10 \%-/ 5 \%-/ 1 \%$-level. Transitions take place between the following labour market states: Permanent employment (PermE), temporary employment, (TempE) unemployment (U), self-employment (S), education (Ed) and inactivity (I); only estimates for the first three categories are presented. - Robust standard errors clustered at country level in parentheses

Table 19 Yearly transitions from unemployment to different labour market states (detailed employment states), age group-specific effects of the crisis

\begin{tabular}{llll}
\hline & $U$ & PermE & TempE \\
\hline Age 15-24 & $-0.099^{c}$ & $0.0288^{c}$ & $0.0703^{c}$ \\
& $(0.0296)$ & $(0.0085)$ & $(0.0116)$ \\
Age 25-34 & $-0.0645^{c}$ & $0.0293^{c}$ & $0.0467^{c}$ \\
& $(0.0182)$ & $(0.0065)$ & $(0.0078)$ \\
Age 35-54 & Reference category & Reference category & Reference category \\
Age 55-65 & 0.0204 & $-0.0659^{c}$ & $-0.1138^{c}$ \\
& $(0.0205)$ & $(0.006)$ & $(0.0159)$ \\
Crisis indicator & 0.0404 & $-0.0202^{c}$ & -0.0102 \\
& $(0.0367)$ & $(0.006)$ & $(0.0151)$ \\
Crisis ${ }^{*}$ Age 15-24 & 0.0051 & -0.0060 & -0.0124 \\
& $(0.0192)$ & $(0.0061)$ & $(0.011)$ \\
Crisis ${ }^{*}$ Age 25-34 & 0.0153 & -0.0040 & -0.0070 \\
& $(0.0114)$ & $(0.0075)$ & $(0.0064)$ \\
Crisis ${ }^{*}$ Age 55-65 & 0.0086 & -0.0001 & -0.0124 \\
& $(0.0247)$ & $(0.0144)$ & $(0.0169)$ \\
Other individual covariates & included & included & included \\
Country dummies & included & included & included \\
Year dummies & included & included & included \\
Pseudo-R-squared & 0.0845 & & \\
Observations & 65,872 & & \\
\hline EU-SILC own cal & & \\
\hline
\end{tabular}

EU-SILC, own calculations. No data available for Denmark and the United Kingdom. Multinomial logit model. a $/ \mathrm{b} / \mathrm{c}$ : statistically significant at least at the 10\%-/5\%-/1\%-level. Transitions take place between the following labour market states: Permanent employment (PermE), temporary employment, (TempE) unemployment (U), self-employment (S), education (Ed) and inactivity (I); only estimates for the first three categories are presented. - Robust standard errors clustered at country level in parentheses 
Table 20 Yearly transitions from unemployment to different labour market states (detailed employment states), skill group-specificeffects of the crisis

\begin{tabular}{|c|c|c|c|}
\hline & $U$ & PermE & TempE \\
\hline \multirow[t]{2}{*}{ Low skilled (ISCED 0-2) } & $0.0503^{b}$ & $-0.0402^{c}$ & $-0.0257^{c}$ \\
\hline & $(0.0203)$ & $(0.0065)$ & $(0.0097)$ \\
\hline Medium skilled (ISCED 3-4) & Reference category & Reference category & Reference category \\
\hline \multirow[t]{2}{*}{ High skilled (ISCED 5) } & $-0.0797^{c}$ & $0.0462^{c}$ & $0.0275^{\mathrm{a}}$ \\
\hline & $(0.0134)$ & $(0.0051)$ & $(0.0157)$ \\
\hline \multirow[t]{2}{*}{ Crisis indicator } & 0.0236 & $-0.0223^{c}$ & -0.0150 \\
\hline & $(0.0232)$ & $(0.008)$ & $(0.0121)$ \\
\hline \multirow[t]{2}{*}{ Crisis $^{*}$ Low skilled } & 0.0349 & 0.0128 & -0.0079 \\
\hline & $(0.0329)$ & $(0.0123)$ & $(0.0135)$ \\
\hline \multirow[t]{2}{*}{ Crisis $^{*}$ High skilled } & $0.041^{\mathrm{a}}$ & $-0.0211^{a}$ & 0.0105 \\
\hline & $(0.0217)$ & $(0.011)$ & (0.0139) \\
\hline Other individual covariates & included & included & included \\
\hline Country dummies & included & included & included \\
\hline Year dummies & included & included & included \\
\hline Pseudo-R-squared & 0.0848 & & \\
\hline Observations & 65,872 & & \\
\hline
\end{tabular}

EU-SILC, own calculations. No data available for Denmark and the United Kingdom. Multinomial logit model. ${ }^{\mathrm{a}} / \mathrm{b} / \mathrm{c}$ : statistically significant at least at the 10\%-/5\%-/1\%-level. Transitions take place between the following labour market states: Permanent employment (PermE), temporary employment, (TempE) unemployment (U), self-employment (S), education (Ed) and inactivity (I); only estimates for the first three categories are presented. - Robust standard errors clustered at country level in parentheses

as a reaction to the crisis are likely to be multi-faceted. Therefore, in the final step of our analysis, we perform an econometric analysis at the level of country clusters. We follow Esping-Andersen (1990) in defining country clusters: The Anglo-Saxon cluster (IE, UK), Scandinavia (DK, FI, IS, NO, SE), Continental Europe (AT, BE, FR, LU, NL), Mediterranean Europe (CY, ES, GR, IT, PT), and Central and Eastern Europe (CEE: BG, CZ, EE, HU, LT, LV, PL, SI, SK). ${ }^{12}$ Despite some institutional variation within these country clusters, the clusters can be viewed as approximations to certain labour market

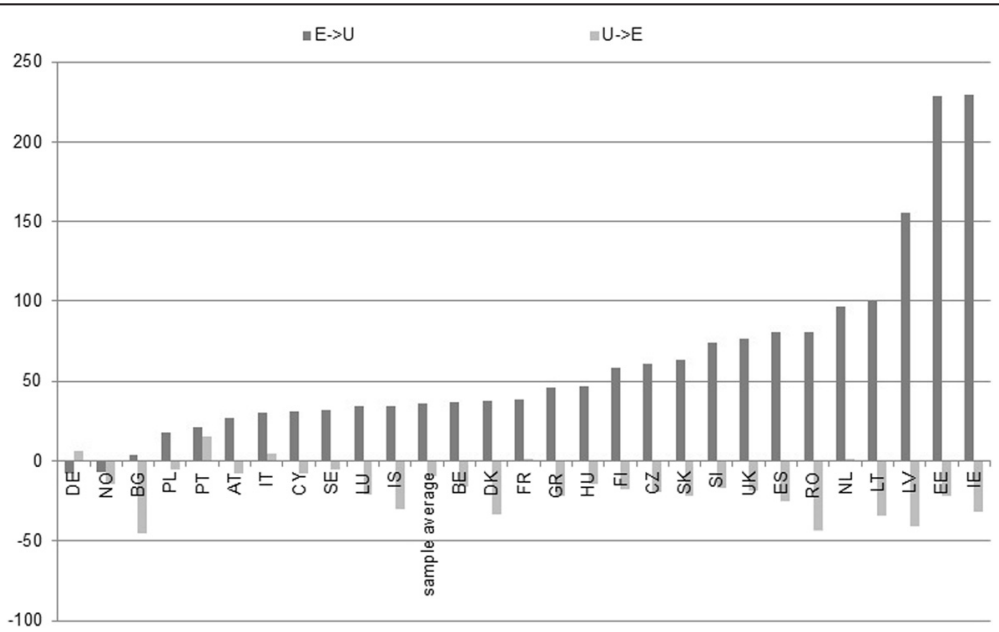

Fig. 2 Flow rates between employment and unemployment: Growth rates between pre-crisis and crisis period by country. Source: EU-LFS (for DE and RO) and EU-SILC, own calculations 


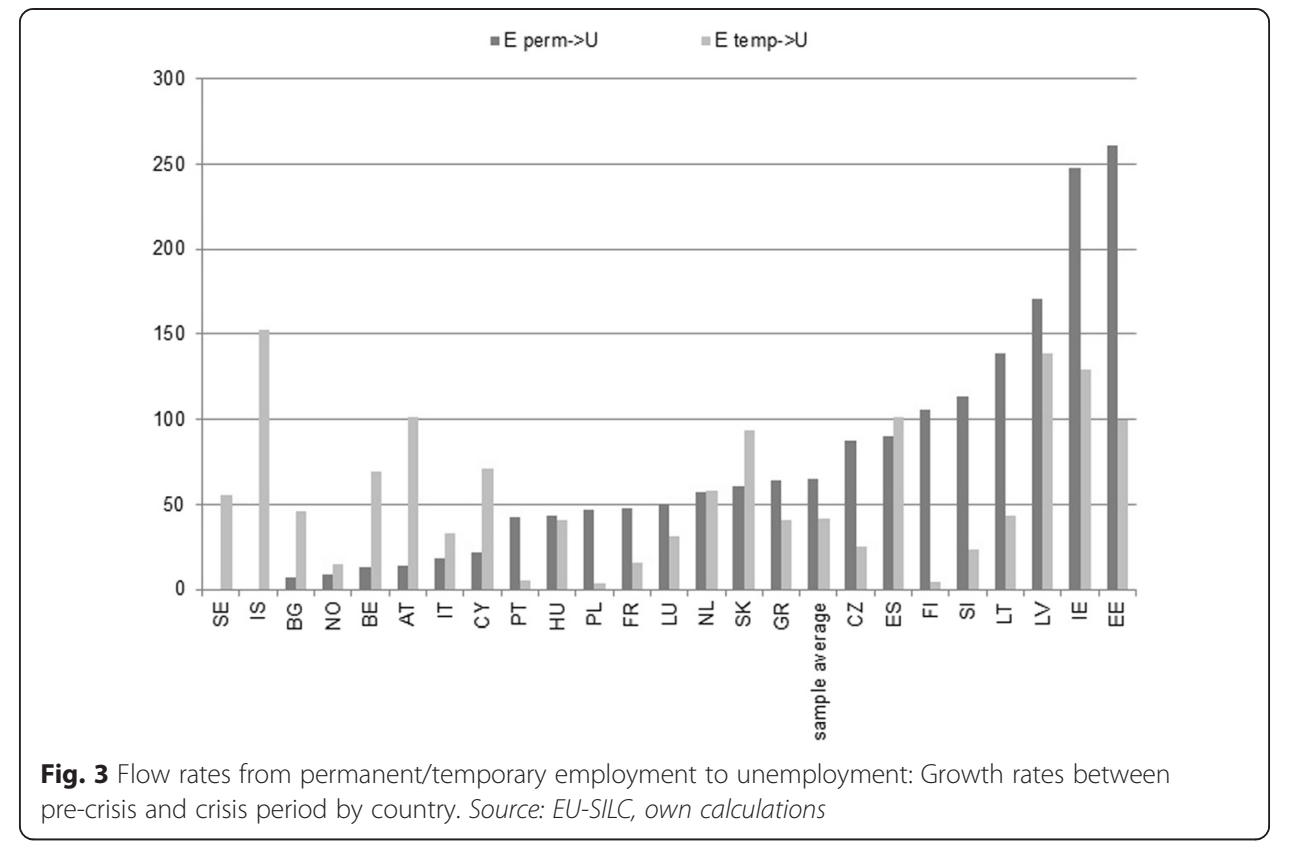

frameworks: the Anglo-Saxon countries stand for very flexible labour markets, Scandinavia for high flexibility and high social security (e.g. unemployment benefits, which are however strictly monitored), Continental Europe for low flexibility and high social security, Mediterranean countries for dual labour markets and low social security, and CEE for high flexibility and low social security (Boeri and van Ours, 2013; Martin and Scarpetta 2012). Examining country groups rather than single institutions allows us to take into account the complementarity of labour market institutions, i.e. the fact that these institutions usually have a joint effect on labour market outcomes.

We thus run a multinomial regression model at the individual level (see Equation 1) and focus on outflows from employment because these transitions were the most affected flows for national labour markets in the majority of countries during the crisis. As the cross-country differences in transitions are strongly influenced by the depth of the recession in the respective countries, we include country-specific GDP growth as an explanatory variable in the econometric model, in addition to the individual-level socio-economic variables and yearly dummies used above (e.g. Table 2). Furthermore, we include dummy variables for the different country clusters in order to capture level differences in transition probabilities which are constant over time. Finally, we add interactions between the crisis indicator variable and the cluster dummies as explanatory variables. Given that we control for GDP growth, these interactions show how labour markets differed in their reaction to the crisis beyond differences which are due to the depth of the recession. Therefore, differences in the interaction coefficients can be attributed to differences in the institutional framework prevailing in the country clusters.

The regression results show that, for all countries considered jointly, GDP growth is significantly correlated with the probability of remaining employed and of making a transition from employment to either unemployment or education, with the latter two correlations being counter-cyclical (Table 21). By contrast, the transitions from employment to self-employment and to inactivity are acyclical. 
Table 21 Yearly transitions from employment to different labour market states, country cluster-specific effects of the crisis

\begin{tabular}{|c|c|c|c|c|c|}
\hline Country cluster & $\mathrm{EE}$ & ES & EU & EEd & El \\
\hline \multirow[t]{2}{*}{ GDP growth } & $0.00118^{c}$ & -0.00014 & $-0.00081^{c}$ & $-0.00002^{b}$ & -0.00021 \\
\hline & $(0.00026)$ & $(0.00013)$ & $(0.00017)$ & $(0.00001)$ & $(0.00021)$ \\
\hline Anglo-Saxon & Reference & Reference & Reference & Reference & Reference \\
\hline \multirow[t]{2}{*}{ Scandinavian } & $-0.0191^{\mathrm{a}}$ & $-0.0035^{c}$ & $0.0170^{\mathrm{a}}$ & $-0.0021^{c}$ & -0.0035 \\
\hline & $(0.0112)$ & $(0.0012)$ & $(0.0088)$ & $(0.0003)$ & $(0.0022)$ \\
\hline \multirow[t]{2}{*}{ Continental } & $-0.0143^{a}$ & $-0.0049^{b}$ & $0.0247^{c}$ & $-0.0007^{c}$ & $-0.0049^{b}$ \\
\hline & $(0.0074)$ & $(0.0021)$ & $(0.0086)$ & $(0.0002)$ & $(0.0021)$ \\
\hline \multirow[t]{2}{*}{ Mediterranean } & $-0.0441^{c}$ & $0.0106^{c}$ & $0.0340^{\mathrm{b}}$ & $-0.0007^{c}$ & 0.0002 \\
\hline & $(0.0097)$ & $(0.0023)$ & $(0.0133)$ & $(0.0002)$ & $(0.0022)$ \\
\hline \multirow[t]{2}{*}{ CEE } & $-0.0536^{c}$ & $0.0061^{b}$ & $0.0419^{c}$ & $-0.0008^{c}$ & $0.0064^{\mathrm{a}}$ \\
\hline & $(0.0096)$ & $(0.0025)$ & $(0.0084)$ & $(0.0001)$ & $(0.0037)$ \\
\hline \multirow[t]{2}{*}{ Crisis $^{*}$ Anglo-Saxon } & $-0.0231^{c}$ & $0.0012^{\mathrm{a}}$ & $0.0180^{c}$ & -0.0001 & $0.0040^{c}$ \\
\hline & $(0.0034)$ & $(0.0007)$ & $(0.0034)$ & $(0.0001)$ & $(0.0009)$ \\
\hline \multirow[t]{2}{*}{ Crisis ${ }^{*}$ Scandinavian } & -0.0016 & -0.0009 & $0.0085^{c}$ & -0.0002 & $-0.0058^{c}$ \\
\hline & $(0.0045)$ & $(0.0020)$ & $(0.0025)$ & $(0.0001)$ & $(0.0019)$ \\
\hline \multirow[t]{2}{*}{ Crisis $^{*}$ Continental } & -0.0046 & 0.0003 & $0.0064^{c}$ & -0.0001 & $-0.0020^{c}$ \\
\hline & $(0.0029)$ & $(0.0014)$ & $(0.0012)$ & $(0.0001)$ & $(0.0008)$ \\
\hline \multirow[t]{2}{*}{ Crisis $^{*}$ Mediterranean } & -0.0051 & $-0.0024^{c}$ & 0.0090 & 0.0001 & -0.0015 \\
\hline & $(0.0043)$ & $(0.0008)$ & $(0.0058)$ & $(0.0001)$ & $(0.0013)$ \\
\hline \multirow[t]{2}{*}{ Crisis $^{*}$ CEE } & -0.0067 & 0.0015 & $0.0082^{c}$ & $-0.0004^{c}$ & -0.0026 \\
\hline & $(0.0055)$ & $(0.0026)$ & $(0.0030)$ & $(0.0001)$ & $(0.0017)$ \\
\hline Individual covariates & included & included & included & Included & included \\
\hline Year dummies & included & included & included & included & included \\
\hline Pseudo-R-squared & 0.1003 & & & & \\
\hline Observations & 578,331 & & & & \\
\hline
\end{tabular}

EU-SILC, own calculations. Multinomial logit model. ${ }^{\mathrm{a}} /^{\mathrm{b}} / \mathrm{c}$ : statistically significant at least at the 10\%-/5\%-/1\%-level. - The model includes transitions between the following labour market states: employment (E) (i.e. persons remaining in employment), unemployment $(\mathrm{U})$, self-employment $(\mathrm{S})$, education (Ed) and inactivity (I). - Robust standard errors clustered at the country level in parentheses

Turning to cluster-specific effects, it becomes apparent that, net of GPD growth, the Mediterranean and CEE clusters displayed a higher employment stability than the reference cluster (Anglo-Saxon) before the crisis. Furthermore, transitions from employment to unemployment were significantly lower in the Anglo-Saxon cluster than in all the other clusters. Finally, the Continental cluster displayed a remarkably lower transition probability from employment to inactivity than the other country clusters.

During the crisis, employment stability declined particularly strongly in the AngloSaxon country cluster, as the coefficients on the interaction of the crisis dummy with the country cluster dummies make clear. In the other country clusters, the transition probability evolved in line with the development of country-specific GDP and yearly European trends. Turning to transitions from employment to unemployment, the results indicate that the Anglo-Saxon cluster again displays the strongest labour-market reaction, i.e. a strongly above-average increase in the respective transition rate. In the Mediterranean, Scandinavian and CEE clusters, the EU transition probability also increased disproportionately, but to a smaller extent than in the Anglo-Saxon cluster. ${ }^{13}$ 
Finally, during the crisis the transition probability to inactivity rose disproportionately in the Anglo-Saxon country cluster, while the opposite was the case in the Continental cluster and, especially, the Scandinavian cluster.

As argued above, these results are likely to be mainly driven by the institutional framework prevailing in the different country clusters. In this context, it is important to point out that our analysis focuses on the extensive margin. Changes along the intensive margin and/or wage adjustments constitute alternative adjustment patterns. While labour market institutions always exert a joint effect on labour market outcomes, employment protection plays a particularly important role in this context. As stressed by Cazes et al. (2013), countries with low employment protection feature more external flexibility and less internal flexibility. The UK is an example for such an economy, which can at least partly explain the above result of a strong increase in flows from employment to unemployment during the Great Recession. By contrast, the Mediterranean country cluster includes countries such as Italy, Portugal and Spain with relatively high employment protection. Consequently, this cluster features employment-to-unemployment transitions which are in line with its GDP development and aggregate European trends.

\section{Conclusions}

Using the individual-level EU-SILC and EU-LFS data, we examine the labour market transitions in Europe and the effects of the recent financial and economic crisis, highlighting differences between socio-demographic groups and employment types. Our main findings can be summarised as follows. First, the crisis in its early phase (2008-2010) predominantly affected transitions from employment to unemployment and vice versa. The other labour market transitions remained virtually unchanged.

Second, we reveal heterogeneities in the evolution of labour market transitions: The increase in the transition rate from employment to unemployment was particularly pronounced for young persons, medium-skilled persons, and for men. The transition rate from unemployment to employment, on the other hand, fell more strongly for men than for women during the Great Recession. Furthermore, our results indicate that unemployment became more persistent for the high-skilled during the crisis.

Third, temporary contracts played a prominent role for labour market dynamics during the Great Recession in a number of European countries. In particular, transitions from temporary employment to unemployment increased more strongly during the crisis than transitions from permanent employment to unemployment. This finding holds true especially for men and high-skilled workers, suggesting that temporary contracts drive the gender- and skill-related heterogeneities that we detect. Furthermore, the overall outflow rate from temporary employment remained relatively constant during the crisis. This can be explained by offsetting effects: the transition rate from temporary employment to unemployment increases significantly, while the transition rate from temporary employment to permanent employment declines significantly.

Fourth, inflows into unemployment increased more strongly during the crisis in most countries. Given that we focus on the time period 2005-2010, this result is consistent with the finding of Fujita and Ramey (2009) and Fujita (2011) for the US, stressing the importance of the separation rate during the early phase of the recessionary period.

Furthermore, we observe important country heterogeneities, which the depth of the crisis and country-specific institutional features partly explain. Our results in this 
context focus on the extensive margin, which is likely to be strongly influenced by employment protection. An analysis of alternative adjustment mechanisms, such as wage and working hours adjustments, is clearly warranted, but beyond the scope of this paper.

Two caveats are in order. On the one hand, using yearly data, the time aggregation bias may affect our results since we analyse relatively persistent labour market transitions. While previous research has shown that the cyclical features of labour markets remain relatively unchanged when accounting for time aggregation (Elsby et al. 2009; Nekarda 2009), it is nevertheless conceivable that the time aggregation bias differs between countries. Such differences would affect the level differences in labour market transitions between countries, although not necessarily the cross-country differences in cyclicality. This issue is, however, left for future research. On the other hand, the EU-SILC panel data used in our analysis does not allow us to analyse the sectoral dimension, which could partly explain our results such as the gender differences mentioned above.

Our results have several important policy implications. Our finding of strong heterogeneous effects especially for young workers is particularly worrisome as the literature indicates that an unfavourable career start usually has long-lasting, scarring effects (Kahn 2010; Oreopoulos et al. 2012). This calls for economic policy-making that targets young workers. In addition, temporary employment dropped, and its stepping-stone function into permanent employment dwindled. Despite this, temporary employment remains a port of entry into the labour market from unemployment during the recession. Therefore, our results can be viewed as an indication that temporary employment can contribute to a recovery of overall employment in the aftermath of the crisis-an aspect that should be monitored as the European labour markets recover from the Great Recession.

\section{Endnotes}

${ }^{1}$ Note, however, that we do not perform an unemployment decomposition exercise in the spirit of Shimer (2012) or Hairault et al. (2015) but rather focus on analyses at the individual level.

${ }^{2}$ Except for Croatia.

${ }^{3}$ Ohanian and Raffo (2012) challenge this view and argue that higher labour market frictions make the intensive margin of labour adjustment more important in Europe. However, van Rens (2012) finds no evidence for this conclusion.

${ }^{4}$ Employing annual data leads to a time aggregation bias in transitions, inducing an underestimation of the true labour market mobility due to the missing transitions that occur between two consecutive years. However, empirical research indicates that although the level of labour market mobility is affected by the time aggregation bias, the effect on its cyclicality is quantitatively small (Elsby et al. 2009; Nekarda 2009).

${ }^{5}$ Note that these occupation dummies control for level differences in the transition rates between different occupations over the entire time period analysed.

${ }^{6}$ This procedure was not applicable for Poland and Slovakia. For those two countries we had to rely on the information of the turning point indicator (OECD).

${ }^{7}$ The corresponding Markov transition matrices for individual countries are presented in Table A.1 in Bachmann et al. (2015). 
${ }^{8}$ The corresponding Markov transition matrices for individual countries are presented in Table A.2 in Bachmann et al. (2015).

${ }^{9}$ Results obtained from estimating a multinomial logit regression for the transitions out of inactivity also provide evidence that the crisis indicator does not significantly affect the transitions from inactivity to temporary employment. Regarding the transitions from education to temporary employment a significant decrease during the crisis can be observed (regression results are not shown here but are available from the authors upon request). However, as in absolute terms transitions from unemployment and inactivity make up the larger part of inflows into temporary employment, these results support the statement that during the crisis temporary employment remains a port of entry into the labour market.

${ }^{10}$ Boeri (2011) investigates this issue extensively for the time period before the Great Recession.

${ }^{11}$ It should, however, be pointed out that the transition rate from temporary to permanent employment is likely to be more strongly affected by the time aggregation bias which is imminent in our yearly data. Using monthly data would probably increase the difference between the two growth rates considered.

${ }^{12}$ We can only do so for the countries contained in EU-SILC for a sufficient number of years, i.e. DE and RO are not included in this analysis (see Section 2).

${ }^{13} \mathrm{~A}$ Wald test shows that the coefficients for the Scandinavian, Continental and CEE clusters are not significantly different from each other.

Competing interests

The IZA Journal of European Labor Studies is committed to the IZA Guiding Principles of Research Integrity. The authors declare that they have observed these principles.

\section{Acknowledgments}

We thank Hanna Frings, Bart Hobijn, Tom Krebs, Christopher Pissarides, Yolanda F. Rebollo Sanz and Thepthida Sopraseuth, an anonymous referee, as well as participants of the conference "(European) Labour Markets and the Economic Crisis", the Annual Meeting of the European Society of Population Economics 2014, the Annual Meeting of the European Association for Labor Economists, and the IZA/NBS/CELSI conference on "European Labor Markets and the Great Recession: Adjustment, Transmission, Interactions" for helpful comments and suggestions.

Responsible editor: Martin Kahanec

\section{Author details}

${ }^{1}$ Rheinisch-Westfälisches Institut für Wirtschaftsforschung, Hohenzollernstr. 1-3, 45131 Essen, Germany.

${ }^{2}$ Forschungsinstitut zur Zukunft der Arbeit (Institute for the Study of Labor), Bonn, Germany. ${ }^{3}$ Ruhr-Universität Bochum, Bochum, Germany.

Received: 17 July 2015 Accepted: 14 August 2015

Published online: 25 September 2015

References

Bachmann R, Bauer TK, David P (2010) Labor Market Entry Conditions, Wages and Job Mobility. IZA Discussion Paper \#4965

Bachmann R, Bechara P, Kramer A, Rzepka S (2015) Labour market dynamics and worker heterogeneity during the Great Recession - Evidence from Europe. IZA Discussion Paper \#9233

Bell DN, Blanchflower DG (2011) Young people and the Great Recession. Oxf Rev Econ Policy 27(2):241-267

Bentolila S, Cahuc P, Dolado JJ, Le Barbanchon T (2012) Two-tier labour markets in the great recession: France versus Spain. Econ J 122(562):F155-F187

Boeri T (2011) Institutional reforms and dualism in European labor markets. In: Card D, Ashenfelter O (eds) Handbook of Labor Economics. North-Holland, Amsterdam, pp 1173-1236

Boeri T, van Ours J (2013) The economics of imperfect labour markets. University Press, Princeton, N.J

Cazes S, Verick S, Al Hussami F (2013) Why did unemployment respond so differently to the global financial crisis across countries? Insights from Okun's Law. IZA J Labor Policy 2:10

Darby MR, Haltiwanger JC, Plant MW (1986) The ins and outs of unemployment: The ins win. NBER Working Papers 1997, National Bureau of Economic Research, Inc.

ECB - European Central Bank (2012) Euro area labour markets and the crisis. ECB Occasional Paper Series No. 138 
Eichhorst W, Feil M, Marx P (2010) Crisis, What crisis? Patterns of adaptation in European labor markets. Appl Econ Q Suppl 56(61):29-64

Elsby M, Hobijn B, Sahin A (2013) Unemployment dynamics in the OECD. Rev Econ Stat 95(2):530-548

Elsby M, Michaels R, Solon G (2009) The ins and outs of cyclical unemployment. Am Econ J: Macroecon 1(1):84-110

Engel M, Schaffner S (2012), How to Use the EU-SILC Panel to Analyse Monthly and Hourly Wages. Ruhr Economic Papers \#390. RWI.

Esping-Andersen G (1990) The three worlds of welfare capitalism. Polity Press, Cambridge, UK

Fujita S (2011) Dynamics of worker flows and vacancies: evidence from the sign restriction approach. J Appl Econometr 26:89-121

Fujita S, Ramey G (2009) The cyclicality of separation and job finding rates. Int Econ Rev 50(2):415-430

Hairault J-O, Le Barbanchon T, Sopraseuth T (2015) The cyclicality of the separation and job finding rates in France. Eur Econ Rev 76:60-84

Hall RE (2005) Job loss, job finding, and unemployment in the U.S. economy over the past fifty years. In M. Gertler and K. Rogoff (Eds.), NBER Macroeconomics Annual. Cambridge, MA: The MIT Press.

Kahn LB (2010) The long-term labor market consequences of graduating from college in a bad economy. Labour Econ 17(2):303-316

King RG, Rebelo ST (1999) Resuscitating Real Business Cycles. In: Taylor IJB, Woodford M (eds) Handbook of Macroeconomics, vol 1B. North-Holland, Amsterdam, pp 927-1007

Langot F, Quintero-Rojas C (2008) European vs. American hours worked: assessing the role of the extensive and intensive margins. IZA Discussion Paper \#3846

Martin JP, Scarpetta S (2012) Setting it right: employment protection, labour reallocation and productivity. De Economist 160(2):89-116

Merkl C, Wesselbaum D (2011) Extensive vs. intensive margin in Germany and the United States: any differences). Appl Econ Lett 18(9):805-808

Muravyev A (2010) Evolution of employment protection legislation in the USSR, CIS and Baltic States, 1985-2009. IZA Discussion Paper \#5365

Nekarda CJ (2009) Understanding unemployment dynamics: the role of time aggregation, Working Paper, Federal Reserve Board of Governors

Ohanian LE, Raffo A (2012) Aggregate hours worked in OECD countries: New measurement and implications for business cycles. J Monetary Econ 59(1):40-56

Oreopoulos P, von Wachter T, Heisz A (2012) The short- and long-term career effects of graduating in a recession. Am Econ J: Appl Econ 4(1):1-29

Petrongolo B, Pissarides CA (2008) The ins and outs of European unemployment. Am Econ Rev 98(2):256-262

Shimer R (2005) The cyclical behavior of equilibrium unemployment and vacancies. Am Econ Rev 95(1):25-49

Shimer R (2012) Reassessing the ins and outs of unemployment. Rev Econ Dyn 15(2):127-148

Silva JI, Vázquez-Grenno J (2013) The ins and outs of unemployment in a two-tier labor market. Labour Econ 24:161-169

Smith JC (2011) The ins and outs of UK unemployment. Econ J 121:402-444

Theodossiou I, Zangelidis A (2009) Should I stay or should I go? The effect of gender, education and unemployment on labour market transitions. Labour Econ 16(5):566-577

Van Rens T (2012) How important is the intensive margin of labor adjustment? Discussion of "aggregate hours worked in OECD countries: New measurement and implications for business cycles" by L. Ohanian and A Raffo. J Monetary Econ 59(1):40-56

Verick S (2009) Who is hit hardest during a financial crisis? The vulnerability of young men and women to unemployment in an economic downturn. IZA Discussion Papers \#4359

Ward-Warmedinger M, Macchiarelli C (2014) Transitions in labour market status in EU labour markets. IZA J Eur Labour Stud 3:17

von Wachter T, Bender S (2008) Do Initial Conditions Persist Between Firms? An Analysis of Firm-Entry Cohort Effects and Job Losers using Matched Employer-Employee Data. In: Bender S, Lane J, Shaw K, Andersson F, von Wachter T (eds) The Analysis of Firms and Employees: Quantitative and Qualitative Approaches. University of Chicago Press, Chicago, pp 135-162

Yashiv E (2008) U.S. Labor market dynamics revisited. Scand J Econ 109(4):779-806

\section{Submit your manuscript to a SpringerOpen ${ }^{\circ}$ journal and benefit from:}

- Convenient online submission

- Rigorous peer review

- Immediate publication on acceptance

- Open access: articles freely available online

- High visibility within the field

- Retaining the copyright to your article

Submit your next manuscript at $>$ springeropen.com 